\title{
Transition properties of potassium atom
}

\author{
D. K. Nandy ${ }^{1}$ *, Yashpal Singh ${ }^{1}$, B. P. Shah ${ }^{2}$ and B. K. Sahoo ${ }^{1} \dagger$ \\ ${ }^{1}$ Theoretical Physics Division, Physical Research Laboratory, Ahmedabad-380009, India \\ ${ }^{2}$ Department of Physics, Faculty of Science, The M. S. University of Baroda, Vadodara-390012, India
}

(Dated: Received date; Accepted date)

\begin{abstract}
We report here oscillator strengths, transition rates, branching ratios and lifetimes due to allowed transitions in potassium (K) atom. We evaluate electric dipole (E1) amplitudes using an all order relativistic many-body perturbation method. The obtained results are compared with previously available experimental and theoretical studies. Using the E1 matrix elements mentioned above and estimated from the lifetimes of the $4 P$ states, we determine precise values of static and dynamic polarizabilities for the first five low-lying states in the considered atom. The static polarizabilities of the ground and $4 P$ states in the present work are more precise than the available measurements in these states. Only the present work employs relativistic theory to evaluate polarizabilities in the $3 D$ states for which no experimental results are known to compare with. We also reexamine "magic wavelengths" for the $4 P_{1 / 2} \rightarrow 4 S$ and $4 P_{3 / 2} \rightarrow 4 S$ transitions due to the linearly polarized light which are useful to perform state-insensitive trapping of $\mathrm{K}$ atoms.
\end{abstract}

PACS numbers:

\section{INTRODUCTION}

Potassium $(\mathrm{K})$ atom is one of the suitable alkali atoms whose two stable isotopes are fermions and one of them is boson that makes it a special system for cold atom studies [1, 2]. Studies of its transition properties are very useful in manipulating trapping and cooling of this atom [3 -5]. Especially to find out its "magic wavelengths" at which the differential Stark shifts of a transition become zero [6], accurate knowledge of transition properties are necessary [7, 8]. Sophisticated experiments are generally performed to measure these magic wavelengths [9, 10], however they can also be determined precisely by evaluating differential polarizabilities using accurate relativistic many-body methods [7, 8]. In fact, $\mathrm{K}$ atom is an one valence electron system, whose properties can be evaluated precisely that can ultimately act as benchmark tests for the experimental results. Also, a suitable trapping technique of alkali atoms is very much useful in various fields like in the atomic clock, quantum computing, quantum information etc. experiments. In an optical lattice atomic clock experiment, the atoms are trapped by a periodic potential formed by the applied laser beam [11, 12 for which the trapped atoms interact with the oscillating electric field of the trapping beam that causes the shift in the energy levels inside the atoms. But in order to achieve maximum stability of an atomic clock, it is very crucial to tune the laser beam at a particular wavelength such that there will be no effects to the internal clock transition frequency $6,10,12$. These effects can be estimated by estimating dynamic polarizabilities of the atomic states $[6-8,10,12]$.

Accurate values of transition properties in $\mathrm{K}$ are also

\footnotetext{
*Email: dillip@prl.res.in

†Email: bijaya@prl.res.in
}

useful in the astrophysical studies [13 15]. $\mathrm{K}$ is produced in massive stars after oxygen burning and its isotopes abundances are analyzed in 58 metal-poor stars. In these analysis, theoretical estimate of ratios of abundances of $[\mathrm{K} / \mathrm{Fe}]$ with $[\mathrm{Fe} / \mathrm{H}]$ during the chemical evolution of Galaxy are compared with the observations [16. There are also various astrophysical objects such as L dwarfs, $\mathrm{T}$ dwarfs and irradiated giant planets whose spectra are dominated by absorption lines of many alkali atoms 17-20]. Many telescopes operating at various wavelengths from ultraviolet (UV) to infrared (IR) range are used to study various spectral properties of astronomical objects, but these IR telescopes require information about detailed spectroscopic data of atomic systems such as oscillator strengths, transition probabilities etc. 21 23]. Also, identification of new IR lines will be useful in understanding equilibrium temperature, gravity and abundance for many ultra-cool dwarf stars as well as extra solar giant planets in our Galaxy [24]. Therefore it is always very useful to have enough atomic information on new IR atomic lines based on high precision relativistic calculations, so that they can be used in the analysis of spectral measurements generally carried out by sophisticated IR telescopes.

In this paper, we evaluate electric dipole (E1) matrix elements and determine oscillator strengths, transition probabilities, branching ratios, lifetimes and polarizabilities of many states of K atom. Furthermore, we determine the magic wavelengths due to the linearly polarized light for the $4 P_{1 / 2} \rightarrow 4 S$ and $4 P_{3 / 2} \rightarrow 4 S$ transitions and compare them with the available results.

The rest of the paper is organized as follows: in Sections II and III, we give the theories of transition probability due to allowed transition and linear Stark shift and describe briefly about the method of calculation. In the next section, we present the results and discuss about them. Unless stated otherwise, we use atomic unit (a.u.) for all the physical properties through out the paper. 


\section{THEORY AND METHOD OF CALCULATIONS}

\section{A. Allowed radiative properties}

The transition probability (in $s^{-1}$ ) due to E1 matrix element from atomic state $\left|\Psi_{k}\right\rangle$ to $\left|\Psi_{i}\right\rangle$ is given by

$$
A_{k i}=\frac{2.0261 \times 10^{-6}}{\lambda_{k i}^{3} g_{k}} S_{k i}
$$

where $S_{k i}=\left|\left\langle\Psi_{k}|| D \| \Psi_{i}\right\rangle\right|^{2}$ is the line strength, $\lambda_{k i}$ is the wavelength (in $\mathrm{cm}^{-1}$ ) and $g_{k}=2 J_{k}+1$ is the degeneracy factor for angular momentum $J_{k}$ of the state $\left|\Psi_{k}\right\rangle$.

The relative intensities of radiative transitions are generally estimated by their oscillator strengths that for an allowed transition is given by

$$
f_{k i}=1.4992 \times 10^{-24} A_{k i} \frac{g_{k}}{g_{i}} \lambda_{k i}^{2},
$$

which again for the emission and absorption lines are related as

$$
g_{i} f_{i k}=-g_{k} f_{k i}
$$

The lifetime of the $\left|\Psi_{k}\right\rangle$ state due to the allowed transitions (transition probabilities via other multi-poles are neglected for the considered states in the present atom) is estimated by

$$
\tau_{k}=\frac{1}{\sum_{i} A_{k i}}
$$

The branching ratio of a transition probability from the state $\left|\Psi_{k}\right\rangle$ to a lower state $\left|\Psi_{i}\right\rangle$ is given by

$$
\begin{aligned}
\Gamma_{k i} & =\frac{A_{k i}}{\sum_{l} A_{k l}} \\
& =\tau_{k} A_{k i},
\end{aligned}
$$

where sum over $l$ represents all possible allowed transitions from $\left|\Psi_{k}\right\rangle$.

\section{B. Linear Stark shift}

In the application of external electric field described by a plane electromagnetic wave of angular frequency $\omega$, the energy level of a state $\left|\Psi_{k}\right\rangle$ of an atom shifted by

$$
\Delta E_{k}(\omega)=-\frac{1}{2} \alpha_{k}(\omega) \mathcal{E}^{2}
$$

where $\alpha_{k}$ is the known as polarizability of the $\left|\Psi_{k}\right\rangle$ state and $\mathcal{E}$ is the strength of the applied electric field. In a more general form $\alpha_{k}$ in $M_{J_{k}}$ quantum number independent parameters is expressed as

$$
\alpha_{k}(\omega)=\alpha_{v}^{0}(\omega)+\frac{3 M_{J_{k}}^{2}-J_{k}\left(J_{k}+1\right)}{J_{k}\left(2 J_{k}-1\right)} \alpha_{k}^{2}(\omega),
$$

with the parameters $\alpha_{k}^{0}(\omega)$ and $\alpha_{k}^{2}(\omega)$ are called as scalar and tensor polarizabilities, respectively. They are given in terms of the reduced matrix elements of dipole operator $D$ as

$$
\begin{aligned}
\alpha_{k}^{0}(\omega) & =\frac{1}{3\left(2 J_{k}+1\right)} \sum_{l}\left|\left\langle J_{k}\|D\| J_{l}\right\rangle\right|^{2} \\
& \times\left[\frac{1}{E_{l}-E_{k}+\omega}+\frac{1}{E_{l}-E_{k}-\omega}\right]
\end{aligned}
$$

and

$$
\begin{aligned}
\alpha_{k}^{2}(\omega) & =-2 \sqrt{\frac{5 J_{k}\left(2 J_{k}-1\right)}{6\left(J_{k}+1\right)\left(2 J_{k}+1\right)\left(2 J_{k}+3\right)}} \\
& \times \sum_{l}\left\{\begin{array}{ccc}
J_{k} & 2 & J_{k} \\
1 & J_{l} & 1
\end{array}\right\}(-1)^{J_{k}+J_{l}+1}\left|\left\langle J_{k}\|D\| J_{l}\right\rangle\right|^{2} \\
& \times\left[\frac{1}{E_{l}-E_{k}+\omega}+\frac{1}{E_{l}-E_{k}-\omega}\right]
\end{aligned}
$$

for all the allowed states $\left|\Psi_{l}\right\rangle$ from $\left|\Psi_{k}\right\rangle$.

Magic wavelengths of a transition can be determined by evaluating Stark shifts for both the associated states and finding out $\omega$ values where the shifts are equal for these states. Owing to the fact that these effects are directly proportional to the differential polarizabilities of the associated states, magic wavelengths are finally correspond to finding values of $\omega$ that produces null differential dynamic polarizabilities of the transition.

\section{Method of calculations}

In the coupled-cluster ansatz, we express the atomic states with a closed core and one valence orbital as

$$
\left|\Psi_{k}\right\rangle=e^{T}\left\{1+S_{k}\right\}\left|\Phi_{k}\right\rangle
$$

where initial state is constructed as $\left|\Phi_{k}\right\rangle=a_{k}^{\dagger}\left|\Phi_{0}\right\rangle$ with $\left|\Phi_{0}\right\rangle$ is the mean-field wave function for the closed core obtained by Dirac-Hartree-Fock (DHF) method and $a_{k}^{\dagger}$ represents appending the valence orbital denoted by $k$. Here $T$ and $S_{k}$ are the excitation operators that account correlation effects to all orders by exciting electrons from core orbitals and valence along with core orbitals from the corresponding DHF states, respectively. In the present calculations, only singly and doubly excited configurations are generated using both the $T$ and $S_{k}$ operators in the CCSD method framework; also, important correlation effects involving valence electron are accounted through the $S_{k}$ operator perturbatively in the CCSD(T) method approximation. Discussions on these approaches can be found more elaborately in [25]. The details of single particle construction and active space for the calculation of atomic wave functions for the considered atom are described in our previous work 26.

Following the approach given in [27], we evaluate polarizabilities of the considered states in $\mathrm{K}$ atom by expressing different contributions as

$$
\alpha_{k}^{\lambda}=\alpha_{k}^{\lambda}(c)+\alpha_{k}^{\lambda}(v c)+\alpha_{k}^{\lambda}(v)
$$


TABLE I: Results of attachment energies (EAs) in $\mathrm{cm}^{-1}$ of different states K using DHF, CCSD and CCSD(T) methods. The results are compared with the experimental values given in NIST data table [28] and difference between NIST data and results obtained from the $\operatorname{CCSD}(\mathrm{T})$ method are quoted as $\Delta$ in percentage ( $\%)$. third order many-body perturbation theory (MBPT(3) method) following similar approaches as employed in 29 31. Using the dynamic polarizabilities, we verify the "magic wavelengths" in the $4 P_{1 / 2} \rightarrow 4 S$ and $4 P_{3 / 2} \rightarrow 4 S$ transitions and compare them with previously reported values.

TABLE II: Calculated E1 matrix elements and their line strengths in

\begin{tabular}{lccccc}
\hline \hline State & DHF & CCSD & CCSD(T) & NIST $[28]$ & $\Delta$ a. \\
\hline $4 s^{2} S_{1 / 2}$ & -32370.47 & -35016.98 & -35016.59 & -35009 & 0.02 \\
$4 p^{2} P_{1 / 2}$ & -21006.47 & -22010.66 & -22013.81 & -22025 & 0.05 \\
$4 p^{2} P_{3 / 2}$ & -20959.41 & -21950.59 & -21953.72 & -21967 & 0.06 \\
$5 s^{2} S_{1 / 2}$ & -13407.12 & -13982.93 & -13982.35 & -13983 & 0.004 \\
$3 d^{2} D_{5 / 2}$ & -12747.04 & -13418.72 & -13417.33 & -13475 & 0.44 \\
$3 d^{2} D_{3 / 2}$ & -12744.31 & -13417.27 & -13415.78 & -13473 & 0.41 \\
$5 p^{2} P_{1 / 2}$ & -10012.08 & -10303.07 & -10304.42 & -10308 & 0.03 \\
$5 p^{2} P_{3 / 2}$ & -9995.88 & -10283.56 & -10284.89 & -10290 & 0.05 \\
$4 d^{2} D_{5 / 2}$ & -7206.85 & -7572.74 & -7571.24 & -7613 & 0.53 \\
$4 d^{2} D_{3 / 2}$ & -7205.25 & -7571.88 & -7570.84 & -7612 & 0.53 \\
$6 s^{2} S_{1 / 2}$ & -7338.12 & -7558.71 & -7558.34 & -7559 & 0.01 \\
$4 f^{2} F_{5 / 2}$ & -6859.21 & -6881.92 & -6881.95 & -6882 & 0.00 \\
$4 f^{2} F_{7 / 2}$ & -6859.21 & -6881.92 & -6881.94 & -6882 & 0.00 \\
$6 p^{2} P_{1 / 2}$ & -5881.48 & -6008.02 & -6008.75 & -6011 & 0.04 \\
$6 p^{2} P_{3 / 2}$ & -5874.06 & -5999.25 & -5999.98 & -6002 & 0.03 \\
$5 d^{2} D_{5 / 2}$ & -4596.71 & -4800.51 & -4799.29 & -4825 & 0.51 \\
$5 d^{2} D_{3 / 2}$ & -4595.80 & -4800.46 & -4799.23 & -4824 & 0.51 \\
$7 s^{2} S_{1 / 2}$ & -4627.27 & -4735.10 & -4734.85 & -4736 & 0.02 \\
$5 f^{2} F_{7 / 2}$ & -4390.01 & -4403.00 & -4403.01 & -4403 & 0.00 \\
$5 f^{2} F_{5 / 2}$ & -4390.01 & -4403.00 & -4403.01 & -4403 & 0.00 \\
$5 g^{2} G_{7 / 2}$ & -4389.50 & -4390.90 & -4390.91 & -4392 & 0.02 \\
$5 g^{2} G_{9 / 2}$ & -4389.50 & -4390.90 & -4390.90 & -4392 & 0.02 \\
$7 p^{2} P_{1 / 2}$ & -3871.88 & -3938.52 & -3938.99 & -3940 & 0.02 \\
$7 p{ }^{2} P_{3 / 2}$ & -3867.88 & -3933.84 & -3934.31 & -3935 & 0.02 \\
$6 d^{2} D_{5 / 2}$ & -3177.17 & -3298.89 & -3298.31 & -3314 & 0.47 \\
$6 d^{2} D_{3 / 2}$ & -3176.62 & -3298.69 & -3297.97 & -3314 & 0.48 \\
$8 s^{2} S_{1 / 2}$ & -3182.99 & -3244.03 & -3243.85 & -3245 & 0.03 \\
\hline \hline
\end{tabular}

where superscript $\lambda$ with values 0 and 1 correspond to the scalar and tensor polarizabilities, respectively, and notations $c, v c$ and $v$ in the parentheses represent correlation contributions from core, core-valence and valence orbitals, respectively. It has to be noted that the core contribution to the tensor polarizability is zero.

\section{RESULTS AND DISCUSSION}

We present below first the electron attachment energies (EAs) of valence electrons from the states having different valence orbitals with the closed core $\left[3 p^{6}\right]$. Then we present E1 matrix elements and using them with the experimental energies, we determine transition probabilities and other related transition properties. Also, we evaluate $\alpha_{k}^{\lambda}(v)$ for various states from these E1 matrix elements and experimental energies. Other $\alpha_{k}^{\lambda}(c)$ and $\alpha_{k}^{\lambda}(v c)$ contributions to $\alpha_{k}$, which are found to be smaller in magnitudes compared to $\alpha_{k}^{\lambda}(v)$, are evaluated using a

\begin{tabular}{|c|c|c|c|c|}
\hline \multicolumn{2}{|c|}{ Transition $k \rightarrow i$} & \multirow{2}{*}{$\begin{array}{l}\mathrm{DHF} \\
4.554\end{array}$} & \multirow{2}{*}{$\begin{array}{l}\operatorname{CCSD}(\mathrm{T}) \\
4.131(20)\end{array}$} & \multirow{2}{*}{$\begin{array}{l}S_{k i} \\
17.065(160)\end{array}$} \\
\hline $4 p^{2} P_{1 / 2}$ & $\rightarrow 4 s^{2} S_{1 / 2}$ & & & \\
\hline $4 p^{2} P_{3 / 2}$ & $\rightarrow 4 s^{2} S_{1 / 2}$ & 6.439 & $5.841(20)$ & $34.117(234)$ \\
\hline \multirow[t]{2}{*}{$5 s^{2} S_{1 / 2}$} & $\rightarrow 4 p^{2} P_{3 / 2}$ & 5.658 & $5.524(20)$ & $30.515(221)$ \\
\hline & $\rightarrow 4 p^{2} P_{1 / 2}$ & 3.974 & $3.876(10)$ & $15.023(77)$ \\
\hline $3 d^{2} D_{5 / 2}$ & $\rightarrow 4 p^{2} P_{3 / 2}$ & 11.564 & $10.749(50)$ & $115.54(107)$ \\
\hline \multirow[t]{2}{*}{$3 d^{2} D_{3 / 2}$} & $\rightarrow 4 p^{2} P_{3 / 2}$ & 3.855 & $3.583(20)$ & $12.838(143)$ \\
\hline & $\rightarrow 4 p^{2} P_{1 / 2}$ & 8.596 & $7.988(40)$ & $63.808(639)$ \\
\hline \multirow[t]{3}{*}{$5 p^{2} P_{1 / 2}$} & $\rightarrow 3 d^{2} D_{3 / 2}$ & 8.198 & $7.278(130)$ & $52.97(189)$ \\
\hline & $\rightarrow 5 s^{2} S_{3 / 2}$ & 9.935 & $9.489(10)$ & $90.041(190)$ \\
\hline & $\rightarrow 4 s^{2} S_{5 / 2}$ & 0.312 & $0.282(6)$ & $0.079(0.003)$ \\
\hline \multirow[t]{4}{*}{$5 p^{2} P_{3 / 2}$} & $\rightarrow 3 d^{2} D_{5 / 2}$ & 10.955 & $9.729(150)$ & $94.65(292)$ \\
\hline & $\rightarrow 3 d^{2} D_{3 / 2}$ & 3.655 & $3.242(50)$ & $10.511(324)$ \\
\hline & $\rightarrow 5 s^{2} S_{1 / 2}$ & 14.031 & $13.399(20)$ & $179.533(536)$ \\
\hline & $\rightarrow 4 s^{2} S_{1 / 2}$ & 0.456 & $0.416(6)$ & $0.173(5)$ \\
\hline \multirow[t]{2}{*}{$4 d^{2} D_{5 / 2}$} & $\rightarrow 5 p^{2} P_{3 / 2}$ & 23.116 & $22.842(300)$ & $521.8(137)$ \\
\hline & $\rightarrow 4 p^{2} P_{3 / 2}$ & 1.003 & $0.260(5)$ & $0.067(3)$ \\
\hline \multirow[t]{4}{*}{$4 d^{2} D_{3 / 2}$} & $\rightarrow 5 p^{2} P_{3 / 2}$ & 7.704 & $7.613(100)$ & $57.96(152)$ \\
\hline & $\rightarrow 5 p^{2} P_{1 / 2}$ & 17.173 & $16.969(240)$ & $287.95(814)$ \\
\hline & $\rightarrow 4 p^{2} P_{3 / 2}$ & 0.336 & $0.088(5)$ & $0.008(1)$ \\
\hline & $\rightarrow 4 p^{2} P_{1 / 2}$ & 0.769 & $0.220(5)$ & $0.048(2)$ \\
\hline \multirow[t]{4}{*}{$6 s^{2} S_{1 / 2}$} & $\rightarrow 5 p^{2} P_{3 / 2}$ & 12.656 & $12.470(20)$ & $155.50(50)$ \\
\hline & $\rightarrow 5 p^{2} P_{1 / 2}$ & 8.898 & $8.760(10)$ & $76.738(175)$ \\
\hline & $\rightarrow 4 p^{2} P_{3 / 2}$ & 1.309 & $1.287(10)$ & $1.656(26)$ \\
\hline & $\rightarrow 4 p^{2} P_{1 / 2}$ & 0.925 & $0.909(10)$ & $0.826(18)$ \\
\hline \multirow[t]{4}{*}{$4 f^{2} F_{5 / 2}$} & $\rightarrow 4 d^{2} D_{3 / 2}$ & 25.230 & $25.37(146)$ & $643.8(741)$ \\
\hline & $\rightarrow 4 d^{2} D_{5 / 2}$ & 6.743 & $6.78(38)$ & $46.0(52)$ \\
\hline & $\rightarrow 3 d^{2} D_{3 / 2}$ & 14.112 & $12.41(11)$ & $154.1(27)$ \\
\hline & $\rightarrow 3 d^{2} D_{5 / 2}$ & 3.769 & $3.318(30)$ & $11.01(20)$ \\
\hline \multirow[t]{2}{*}{$4 f^{2} F_{7 / 2}$} & $\rightarrow 4 d^{2} D_{5 / 2}$ & 30.158 & $30.34(170)$ & $923(103)$ \\
\hline & $\rightarrow 3 d^{2} D_{5 / 2}$ & 16.857 & $14.84(12)$ & $220.3(36)$ \\
\hline \multirow[t]{5}{*}{$6 p^{2} P_{1 / 2}$} & $\rightarrow 6 s^{2} S_{1 / 2}$ & 17.195 & $16.613(20)$ & $275.99(66)$ \\
\hline & $\rightarrow 4 d^{2} D_{3 / 2}$ & 16.833 & $14.76(37)$ & $217.9(109)$ \\
\hline & $\rightarrow 3 d^{2} D_{3 / 2}$ & 1.025 & $1.037(10)$ & $1.075(21)$ \\
\hline & $\rightarrow 5 s^{2} S_{1 / 2}$ & 0.873 & $0.906(10)$ & $0.820(18)$ \\
\hline & $\rightarrow 4 s^{2} S_{1 / 2}$ & 0.104 & $0.087(5)$ & $0.008(1)$ \\
\hline \multirow[t]{7}{*}{$6 p^{2} P_{3 / 2}$} & $\rightarrow 6 s^{2} S_{1 / 2}$ & 24.273 & $23.444(20)$ & $549.621(938)$ \\
\hline & $\rightarrow 4 d^{2} D_{3 / 2}$ & 7.502 & $6.57(16)$ & $43.20(210)$ \\
\hline & $\rightarrow 4 d^{2} D_{5 / 2}$ & 22.487 & $19.734(470)$ & $389.4(185)$ \\
\hline & $\rightarrow 3 d^{2} D_{3 / 2}$ & 0.462 & $0.467(3)$ & $0.218(3)$ \\
\hline & $\rightarrow 3 d^{2} D_{5 / 2}$ & 1.387 & $1.389(10)$ & $1.954(28)$ \\
\hline & $\rightarrow 5 s^{2} S_{1 / 2}$ & 1.263 & $1.312(6)$ & $1.721(15)$ \\
\hline & $\rightarrow 4 s^{2} S_{1 / 2}$ & 0.155 & $0.132(6)$ & $0.017(2)$ \\
\hline \multirow[t]{5}{*}{$5 d^{2} D_{5 / 2}$} & $\rightarrow 6 p^{2} P_{3 / 2}$ & 38.150 & $38.72(74)$ & $1499(57)$ \\
\hline & $\rightarrow 4 f^{2} F_{7 / 2}$ & 5.803 & $8.42(10)$ & $70.90(17)$ \\
\hline & $\rightarrow 4 f^{2} F_{5 / 2}$ & 1.297 & $1.883(20)$ & $3.546(75)$ \\
\hline & $\rightarrow 5 p^{2} P_{3 / 2}$ & 2.690 & $1.461(5)$ & $2.134(15)$ \\
\hline & $\rightarrow 4 p^{2} P_{3 / 2}$ & 0.122 & $0.374(5)$ & $0.141(4)$ \\
\hline \multirow[t]{2}{*}{$5 d^{2} D_{3 / 2}$} & $\rightarrow 6 p^{2} P_{3 / 2}$ & 12.712 & $12.90(26)$ & $166.5(67)$ \\
\hline & $\rightarrow 6 p^{2} P_{1 / 2}$ & 28.333 & $28.76(57)$ & $827.3(328)$ \\
\hline
\end{tabular}

Continue... 
TABLE II - continued from previous page.

\begin{tabular}{|c|c|c|c|c|}
\hline \multicolumn{2}{|c|}{ Transition $k \rightarrow i$} & $\mathrm{DHF}$ & $\operatorname{CCSD}(\mathrm{T})$ & $S_{k i}$ \\
\hline \multirow{11}{*}{$7 s^{2} S_{1 / 2}$} & $\rightarrow 4 f^{2} F_{5 / 2}$ & 4.844 & $7.034(90)$ & $49.48(127)$ \\
\hline & $\rightarrow 5 p^{2} P_{3 / 2}$ & 0.899 & $0.490(5)$ & $0.240(5)$ \\
\hline & $\rightarrow 5 p^{2} P_{1 / 2}$ & 2.042 & $1.138(10)$ & $1.295(23)$ \\
\hline & $\rightarrow 4 p^{2} P_{3 / 2}$ & 0.042 & $0.124(5)$ & $0.015(1)$ \\
\hline & $\rightarrow 4 p^{2} P_{1 / 2}$ & 0.105 & $0.264(5)$ & $0.071(3)$ \\
\hline & $\rightarrow 6 p^{2} P_{3 / 2}$ & 22.032 & $21.828(20)$ & $476.46(87)$ \\
\hline & $\rightarrow 6 p^{2} P_{1 / 2}$ & 15.496 & $15.342(10)$ & $235.38(31)$ \\
\hline & $\rightarrow 5 p^{2} P_{3 / 2}$ & 2.624 & $2.563(10)$ & $6.569(51)$ \\
\hline & $\rightarrow 5 p^{2} P_{1 / 2}$ & 1.857 & $1.814(10)$ & $3.291(37)$ \\
\hline & $\rightarrow 4 p^{2} P_{3 / 2}$ & 0.686 & $0.677(6)$ & $0.458(8)$ \\
\hline & $\rightarrow 4 p^{2} P_{1 / 2}$ & 0.485 & $0.479(5)$ & $0.229(5)$ \\
\hline \multirow[t]{3}{*}{$5 f^{2} F_{7 / 2}$} & $\rightarrow 5 d^{2} D_{5 / 2}$ & 56.649 & $56.34(331)$ & $3174(373)$ \\
\hline & $\rightarrow 4 d^{2} D_{5 / 2}$ & 20.464 & $15.84(20)$ & $250.84(63)$ \\
\hline & $\rightarrow 3 d^{2} D_{5 / 2}$ & 6.131 & $5.913(40)$ & $34.963(473)$ \\
\hline \multirow[t]{6}{*}{$5 f^{2} F_{5 / 2}$} & $\rightarrow 5 d^{2} D_{3 / 2}$ & 47.394 & $47.12(289)$ & $2220(272)$ \\
\hline & $\rightarrow 5 d^{2} D_{5 / 2}$ & 12.667 & $12.60(74)$ & $158.7(186)$ \\
\hline & $\rightarrow 4 d^{2} D_{3 / 2}$ & 17.142 & $13.24(17)$ & $175.2(45)$ \\
\hline & $\rightarrow 4 d^{2} D_{5 / 2}$ & 4.576 & $3.541(40)$ & $12.54(28)$ \\
\hline & $\rightarrow 3 d^{2} D_{3 / 2}$ & 5.123 & $4.949(30)$ & $24.49(30)$ \\
\hline & $\rightarrow 3 d^{2} D_{5 / 2}$ & 1.371 & $1.322(10)$ & $1.748(26)$ \\
\hline \multirow[t]{4}{*}{$5 g^{2} G_{7 / 2}$} & $\rightarrow 5 f^{2} F_{5 / 2}$ & 41.688 & $41.79(418)$ & $1746(349)$ \\
\hline & $\rightarrow 5 f^{2} F_{7 / 2}$ & 8.019 & $8.04(80)$ & $64.7(128)$ \\
\hline & $\rightarrow 4 f^{2} F_{7 / 2}$ & 6.313 & $6.261(10)$ & $39.20(12)$ \\
\hline & $\rightarrow 4 f^{2} F_{5 / 2}$ & 32.803 & $32.53(16)$ & $1058.4(104)$ \\
\hline \multirow[t]{2}{*}{$5 g^{2} G_{9 / 2}$} & $\rightarrow 5 f^{2} F_{7 / 2}$ & 47.441 & $47.58(480)$ & $2264(457)$ \\
\hline & $\rightarrow 4 f^{2} F_{7 / 2}$ & 37.348 & $37.040(20)$ & $1372(15)$ \\
\hline \multirow[t]{7}{*}{$7 p^{2} P_{1 / 2}$} & $\rightarrow 7 s^{2} S_{1 / 2}$ & 26.351 & $25.595(20)$ & $655.1(10)$ \\
\hline & $\rightarrow 5 d^{2} D_{3 / 2}$ & 27.927 & $24.46(68)$ & $598(33)$ \\
\hline & $\rightarrow 6 s^{2} S_{1 / 2}$ & 1.658 & $1.735(10)$ & $3.010(34)$ \\
\hline & $\rightarrow 4 d^{2} D_{3 / 2}$ & 2.313 & $2.424(30)$ & $5.876(145)$ \\
\hline & $\rightarrow 3 d^{2} D_{3 / 2}$ & 0.484 & $0.500(6)$ & $0.250(6)$ \\
\hline & $\rightarrow 5 s^{2} S_{1 / 2}$ & 0.320 & $0.341(5)$ & $0.460(3)$ \\
\hline & $\rightarrow 4 s^{2} S_{1 / 2}$ & 0.053 & $0.041(5)$ & $0.0020(4)$ \\
\hline \multirow[t]{10}{*}{$7 p^{2} P_{3 / 2}$} & $\rightarrow 7 s^{2} S_{1 / 2}$ & 37.187 & $36.107(20)$ & $1303.7(14)$ \\
\hline & $\rightarrow 5 d^{2} D_{3 / 2}$ & 12.445 & $10.89(36)$ & $118.50(784)$ \\
\hline & $\rightarrow 5 d^{2} D_{5 / 2}$ & 37.305 & $32.69(90)$ & $1068.5(588)$ \\
\hline & $\rightarrow 6 s^{2} S_{1 / 2}$ & 2.389 & $2.501(10)$ & $6.255(50)$ \\
\hline & $\rightarrow 4 d^{2} D_{3 / 2}$ & 1.041 & $1.089(10)$ & $1.186(22)$ \\
\hline & $\rightarrow 4 d^{2} D_{5 / 2}$ & 3.126 & $3.259(40)$ & $10.62(26)$ \\
\hline & $\rightarrow 3 d^{2} D_{3 / 2}$ & 0.218 & $0.225(5)$ & $0.051(2)$ \\
\hline & $\rightarrow 3 d^{2} D_{5 / 2}$ & 0.656 & $0.676(10)$ & $0.587(13)$ \\
\hline & $\rightarrow 5 s^{2} S_{1 / 2}$ & 0.469 & $0.499(5)$ & $0.249(5)$ \\
\hline & $\rightarrow 4 s^{2} S_{1 / 2}$ & 0.081 & $0.064(5)$ & $0.004(1)$ \\
\hline \multirow[t]{8}{*}{$6 d^{2} D_{5 / 2}$} & $\rightarrow 7 p^{2} P_{3 / 2}$ & 56.587 & $58.30(141)$ & $3399(164)$ \\
\hline & $\rightarrow 5 f^{2} F_{5 / 2}$ & 2.843 & $4.070(60)$ & $16.57(49)$ \\
\hline & $\rightarrow 5 f^{2} F_{7 / 2}$ & 12.713 & $18.20(26)$ & $331.39(946)$ \\
\hline & $\rightarrow 6 p^{2} P_{3 / 2}$ & 4.838 & $2.993(20)$ & $8.958(120)$ \\
\hline & $\rightarrow 4 f^{2} F_{5 / 2}$ & 0.416 & $0.541(5)$ & $0.293(5)$ \\
\hline & $\rightarrow 4 f^{2} F_{7 / 2}$ & 1.862 & $2.421(10)$ & $5.861(48)$ \\
\hline & $\rightarrow 5 p^{2} P_{3 / 2}$ & 0.818 & $0.045(5)$ & $0.0020(4)$ \\
\hline & $\rightarrow 4 p{ }^{2} P_{3 / 2}$ & 0.053 & $0.404(5)$ & $0.163(4)$ \\
\hline \multirow[t]{7}{*}{$6 d^{2} D_{3 / 2}$} & $\rightarrow 7 p^{2} P_{3 / 2}$ & 18.854 & $19.43(48)$ & $377.6(186)$ \\
\hline & $\rightarrow 7 p^{2} P_{1 / 2}$ & 42.018 & $43.31(104)$ & $1876(90)$ \\
\hline & $\rightarrow 5 f^{2} F_{5 / 2}$ & 10.614 & $15.22(23)$ & $232(7)$ \\
\hline & $\rightarrow 6 p^{2} P_{3 / 2}$ & 1.617 & $1.002(5)$ & $1.004(10)$ \\
\hline & $\rightarrow 6 p^{2} P_{1 / 2}$ & 3.661 & $2.304(20)$ & $5.308(92)$ \\
\hline & $\rightarrow 4 f^{2} F_{5 / 2}$ & 1.555 & $2.029(20)$ & $4.117(81)$ \\
\hline & $\rightarrow 5 p^{2} P_{3 / 2}$ & 0.275 & $0.015(5)$ & $0.0002(1)$ \\
\hline
\end{tabular}

Continue...
TABLE II - continued from previous page.

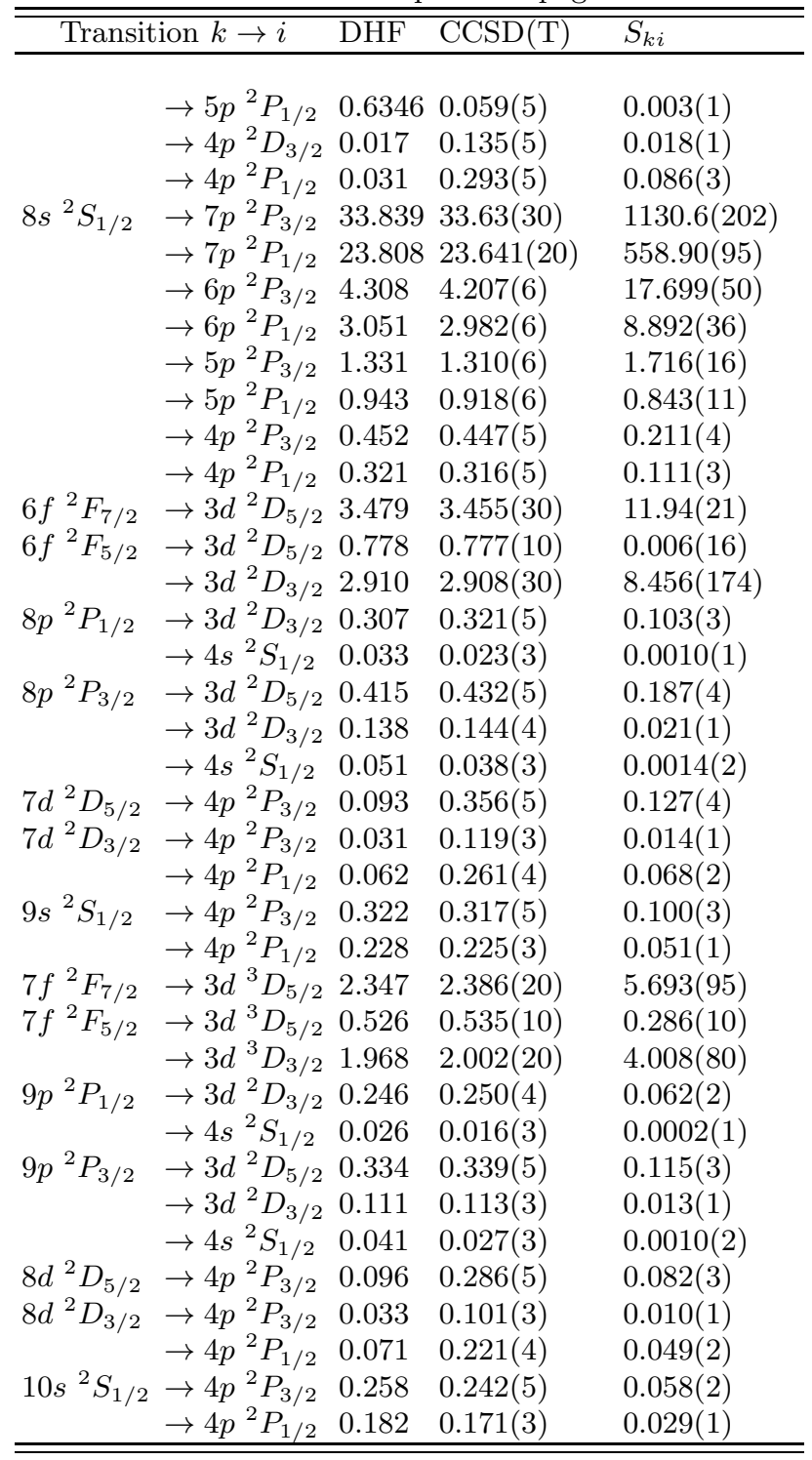

\section{A. Electron attachment energies}

In Table I, we present EAs corresponding to many states using our DHF, CCSD and CCSD(T) methods and they are compared with the corresponding values given by National Institute of Science and Technology (NIST) 28. We consider results obtained from the $\operatorname{CCSD}(\mathrm{T})$ method are the final calculated results. In the above table, we also present differences between results from the $\mathrm{CCSD}(\mathrm{T})$ method and quoted by NIST in percentage as $\Delta$. As seen in the table, all the calculated results are subone percent accurate. Among all other states, results of the $D$ states have large differences with the NIST values. Also, there are large differences between the results obtained using DHF and CCSD(T) methods which indicate the amount of correlation effects involved to determine them. The differences between the results obtained 
from the CCSD and $\operatorname{CCSD}(\mathrm{T})$ methods are small implying contributions from the triple excitations are not very much significant in these calculations. Although the calculated EAs seem to be promising for their sub- one percent accuracies, but we consider the experimental energies wherever required to determine other physical properties in order to minimize uncertainties in the estimated results.

TABLE III: Wavelengths ( $\lambda$ in $\AA$ ), transition rates $\left(A\right.$ in $s^{-1}$ ), oscillator strengths $(f)$ and branching ratios $(\Gamma)$ from different works. The values given in square bracket represent power of 10.

\begin{tabular}{|c|c|c|c|c|c|c|c|}
\hline \multirow{2}{*}{$\begin{array}{l}\text { Upper } \\
\text { state }(f)\end{array}$} & \multirow{2}{*}{$\begin{array}{c}\text { Lower } \\
\text { state }(i)\end{array}$} & \multirow[t]{2}{*}{$\lambda_{k i}$} & \multicolumn{2}{|c|}{$A_{k i}$} & \multicolumn{2}{|l|}{$\overline{\overline{f_{k i}}}$} & \multirow{2}{*}{$\begin{array}{c}\Gamma_{k i} \\
\text { Present }\end{array}$} \\
\hline & & & Others & Present & Others & Present & \\
\hline $4 p_{1 / 2}$ & $\rightarrow 4 s_{1 / 2}$ & 7701.08 & & $3.785[7]$ & $2.93[-1]^{a}$ & $3.34[-1]$ & 1.0 \\
\hline $4 p_{3 / 2}$ & $\rightarrow 4 s_{1 / 2}$ & 7666.99 & & $3.834[7]$ & $5.88[-1]^{a}$ & $6.72[-1]$ & $\sim 1.0$ \\
\hline \multirow[t]{2}{*}{$5 s_{1 / 2}$} & $\rightarrow 4 p_{3 / 2}$ & 12525.58 & $1.58[7]^{b}$ & $1.573[7]$ & $1.72[-1]^{a}, 1.86[-1]^{b}$ & $1.84[-1]$ & 0.665 \\
\hline & $\rightarrow 4 p_{1 / 2}$ & 12435.70 & $7.95[6]^{b}$ & $7.914[6]$ & $1.71[-1]^{a}, 1.84[-1]^{b}$ & $1.82[-1]$ & 0.335 \\
\hline $3 d_{5 / 2}$ & $\rightarrow 4 p_{3 / 2}$ & 11776.10 & $2.38[7]^{b}$ & $2.390[7]$ & $7.69[-1]^{a}, 7.42[-1]^{b}$ & $7.40[-1]$ & $\sim 1.0$ \\
\hline \multirow{2}{*}{$3 d_{3 / 2}$} & $\rightarrow 4 p_{3 / 2}$ & 11772.89 & $3.97[6]^{b}$ & $3.985[6]$ & $8.54[-1]^{a}, 8.24[-1]^{b}$ & $8.20[-1]$ & 0.165 \\
\hline & $\rightarrow 4 p_{1 / 2}$ & 11693.44 & $2.01[7]^{b}$ & $2.021[7]$ & $8.55[-1]^{a}, 8.25[-1]^{b}$ & $8.24[-1]$ & 0.835 \\
\hline \multirow[t]{3}{*}{$5 p_{1 / 2}$} & $\rightarrow 3 d_{3 / 2}$ & 31601.63 & $1.65[6]^{b}$ & $1.700[6]$ & $1.31[-1]^{a}, 1.23[-1]^{b}$ & $1.26[-1]$ & 0.228 \\
\hline & $\rightarrow 5 s_{1 / 2}$ & 27212.13 & & $4.535[6]$ & $4.87[-1]^{a}$ & $4.99[-1]$ & 0.608 \\
\hline & $\rightarrow 4 s_{1 / 2}$ & 4048.36 & & $1.214[6]$ & $2.48[-3]^{a}$ & $2.96[-3]$ & 0.163 \\
\hline \multirow[t]{4}{*}{$5 p_{3 / 2}$} & $\rightarrow 3 d_{3 / 2}$ & 31392.64 & $1.66[5]^{b}$ & $1.717[5]$ & $2.46[-2]^{b}$ & $2.52[-2]$ & 0.022 \\
\hline & $\rightarrow 3 d_{5 / 2}$ & 31415.32 & $1.50[6]^{b}$ & $1.550[6]$ & $1.48[-1]^{b}$ & $1.52[-1]$ & 0.203 \\
\hline & $\rightarrow 5 s_{1 / 2}$ & 27073.93 & & $4.582[6]$ & $9.76[-1]^{a}$ & 1.001 & 0.601 \\
\hline & $\rightarrow 4 s_{1 / 2}$ & 4045.28 & & $1.324[6]$ & $5.40[-3]^{a}$ & $6.48[-3]$ & 0.174 \\
\hline \multirow[t]{2}{*}{$4 d_{5 / 2}$} & $\rightarrow 5 p_{3 / 2}$ & 37356.22 & & $3.380[6]$ & $1.09^{a}$ & 1.054 & 0.981 \\
\hline & $\rightarrow 4 p_{3 / 2}$ & 6966.61 & $1.37[4]^{b}$ & $6.751[4]$ & $2.56[-4]^{a}, 1.49[-4]^{b}$ & $7.324[-4]$ & 0.019 \\
\hline \multirow[t]{4}{*}{$4 d_{3 / 2}$} & $\rightarrow 5 p_{3 / 2}$ & 37341.30 & & $5.638[5]$ & $1.22[-1]^{a}$ & $1.17[-1]$ & 0.161 \\
\hline & $\rightarrow 5 p_{1 / 2}$ & 37081.53 & & $2.860[6]$ & $1.22[-1]^{a}$ & $1.17[-1]$ & 0.815 \\
\hline & $\rightarrow 4 p_{3 / 2}$ & 6966.09 & $2.40[3]^{b}$ & $1.160[4]$ & $2.96[-5]^{a}, 1.75[-5]^{b}$ & $8.39[-5]$ & 0.003 \\
\hline & $\rightarrow 4 p_{1 / 2}$ & 6938.20 & $1.90[4]^{b}$ & $7.340[4]$ & $4.19[-4]^{a}, 2.75[-4]^{b}$ & $1.05[-3]$ & 0.021 \\
\hline \multirow[t]{4}{*}{$6 s_{1 / 2}$} & $\rightarrow 5 p_{3 / 2}$ & 36622.39 & & $3.207[6]$ & $3.17[-1]^{a}$ & $3.20[-1]$ & 0.259 \\
\hline & $\rightarrow 5 p_{1 / 2}$ & 36372.50 & & $1.615[6]$ & $3.15[-1]^{a}$ & $3.18[-1]$ & 0.131 \\
\hline & $\rightarrow 4 p_{3 / 2}$ & 6940.68 & $4.95[6]^{b}$ & $5.019[6]$ & $1.79[-2]^{b}$ & $1.80[-2]$ & 0.406 \\
\hline & $\rightarrow 4 p_{1 / 2}$ & 6912.99 & $2.50[6]^{b}$ & $2.534[6]$ & $1.71[-2]^{a}, 1.79[-2]^{b}$ & $1.80[-2]$ & 0.205 \\
\hline \multirow[t]{4}{*}{$4 f_{5 / 2}$} & $\rightarrow 4 d_{3 / 2}$ & 137040.74 & & $8.457[4]$ & $3.70[-1]^{a}$ & $3.54[-1]$ & 0.005 \\
\hline & $\rightarrow 4 d_{5 / 2}$ & 136841.40 & & $6.063[3]$ & $1.76[-2]^{a}$ & $1.69[-2]$ & 0.0004 \\
\hline & $\rightarrow 3 d_{3 / 2}$ & 15172.52 & $1.45[7]^{b}$ & $1.490[7]$ & $8.24[-1]^{a}, 7.51[-1]^{b}$ & $7.66[-1]$ & 0.928 \\
\hline & $\rightarrow 3 d_{5 / 2}$ & 15167.21 & $1.04[6]^{b}$ & $1.065[6]$ & $3.92[-2]^{a}, 3.57[-2]^{b}$ & $3.65[-2]$ & 0.066 \\
\hline \multirow[t]{2}{*}{$4 f_{7 / 2}$} & $\rightarrow 4 d_{5 / 2}$ & 136840.10 & & $9.097[4]$ & $3.53[-1]^{a}$ & $3.38[-1]$ & 0.006 \\
\hline & $\rightarrow 3 d_{5 / 2}$ & 15167.21 & $1.55[7]^{b}$ & $1.599[7]$ & $7.85[-1]^{a}, 7.15[-1]^{b}$ & $7.31[-1]$ & 0.994 \\
\hline \multirow[t]{5}{*}{$6 p_{1 / 2}$} & $\rightarrow 6 s_{1 / 2}$ & 64576.12 & & $1.038[6]$ & $6.41[-1]^{a}$ & $6.45[-1]$ & 0.345 \\
\hline & $\rightarrow 4 d_{3 / 2}$ & 62456.16 & & $9.061[5]$ & $2.61[-1]^{a}$ & $2.63[-1]$ & 0.301 \\
\hline & $\rightarrow 3 d_{3 / 2}$ & 13400.73 & $4.47[5]^{b}$ & $4.527[5]$ & $6.48[-3]^{a}, 6.02[-3]^{b}$ & $6.06[-3]$ & 0.151 \\
\hline & $\rightarrow 5 s_{1 / 2}$ & 12542.77 & & $4.214[5]$ & $9.42[-3]^{a}$ & $9.88[-3]$ & 0.140 \\
\hline & $\rightarrow 4 s_{1 / 2}$ & 3448.36 & & $1.871[5]$ & $2.51[-4]^{a}$ & $3.31[-4]$ & 0.062 \\
\hline \multirow[t]{7}{*}{$6 p_{3 / 2}$} & $\rightarrow 6 s_{1 / 2}$ & 64226.09 & & $1.051[6]$ & $1.28^{a}$ & 1.292 & 0.341 \\
\hline & $\rightarrow 4 d_{3 / 2}$ & 62128.66 & & $9.125[4]$ & $5.21[-2]^{a}$ & $5.25[-2]$ & 0.030 \\
\hline & $\rightarrow 4 d_{5 / 2}$ & 62087.39 & & $8.242[5]$ & $3.12[-1]^{a}$ & $3.16[-1]$ & 0.267 \\
\hline & $\rightarrow 3 d_{3 / 2}$ & 13385.59 & $4.54[4]^{b}$ & $4.606[4]$ & $1.31[-3]^{a}, 1.22[-3]^{b}$ & $1.23[-3]$ & 0.149 \\
\hline & $\rightarrow 3 d_{5 / 2}$ & 13381.45 & $4.54[4]^{b}$ & $4.131[5]$ & $7.88[-3]^{a}, 7.36[-3]^{b}$ & $7.35[-3]$ & 0.134 \\
\hline & $\rightarrow 5 s_{1 / 2}$ & 12529.51 & & $4.433[5]$ & $1.98[-2]^{a}$ & $2.07[-2]$ & 0.144 \\
\hline & $\rightarrow 4 s_{1 / 2}$ & 3447.36 & & $2.154[5]$ & $5.91[-4]^{a}$ & $7.63[-4]$ & 0.070 \\
\hline \multirow[t]{5}{*}{$5 d_{5 / 2}$} & $\rightarrow 6 p_{3 / 2}$ & 84923.52 & & $8.267[5]$ & $1.380^{a}$ & 1.332 & 0.590 \\
\hline & $\rightarrow 4 f_{7 / 2}$ & 48605.27 & & $2.085[5]$ & $6.10[-2]^{a}$ & $5.50[-2]$ & 0.150 \\
\hline & $\rightarrow 4 f_{5 / 2}$ & 48605.27 & & $1.043[4]$ & $4.07[-3]^{a}$ & $3.67[-3]$ & 0.007 \\
\hline & $\rightarrow 5 p_{3 / 2}$ & 18297.93 & & $1.176[5]$ & $6.10[-3]^{a}$ & $8.80[-3]$ & 0.084 \\
\hline & $\rightarrow 4 p_{3 / 2}$ & 5833.51 & $3.71[5]^{b}$ & $2.379[5]$ & $2.47[-3]^{a}, 2.84[-3]^{b}$ & $1.81[-3]$ & 0.170 \\
\hline \multirow[t]{3}{*}{$5 d_{3 / 2}$} & $\rightarrow 6 p_{3 / 2}$ & 84886.93 & & $1.379[5]$ & $1.53[-1]^{a}$ & $1.48[-1]$ & 0.098 \\
\hline & $\rightarrow 6 p_{1 / 2}$ & 84283.10 & & $6.999[5]$ & $1.530^{a}$ & 1.482 & 0.510 \\
\hline & $\rightarrow 4 f_{5 / 2}$ & 48593.28 & & $2.184[5]$ & $5.69[-2]^{a}$ & $5.12[-2]$ & 0.156 \\
\hline
\end{tabular}


TABLE III - continued from previous page.

\begin{tabular}{|c|c|c|c|c|c|c|c|}
\hline \multirow{2}{*}{$\begin{array}{l}\text { Upper } \\
\text { state }(f\end{array}$} & \multirow{2}{*}{$\begin{array}{c}\text { Lower } \\
\text { state }(i)\end{array}$} & \multirow[t]{2}{*}{$\lambda_{k i}$} & \multicolumn{2}{|c|}{$A_{k i}$} & \multicolumn{2}{|l|}{$f_{k i}$} & \multirow{2}{*}{$\begin{array}{c}\Gamma_{k i} \\
\text { Present }\end{array}$} \\
\hline & & & Others & Present & Others & Present & \\
\hline \multirow{10}{*}{$7 s_{1 / 2}$} & $\rightarrow 5 p_{3 / 2}$ & 18296.22 & & $1.986[4]$ & $6.83[-4]^{a}$ & $9.90[-4]$ & 0.014 \\
\hline & $\rightarrow 5 p_{1 / 2}$ & 18233.65 & & $1.082[5]$ & $7.49[-3]^{a}$ & $1.07[-2]$ & 0.077 \\
\hline & $\rightarrow 4 p_{3 / 2}$ & 5833.33 & $6.13[4]^{b}$ & $3.924[4]$ & $2.72[-4]^{a}, 3.13[-4]^{b}$ & $1.99[-4]$ & 0.028 \\
\hline & $\rightarrow 4 p_{1 / 2}$ & 5813.76 & $2.86[5]^{b}$ & $1.796[5]$ & $2.52[-3]^{a}, 2.90[-3]^{b}$ & $1.81[-3]$ & 0.128 \\
\hline & $\rightarrow 6 p_{3 / 2}$ & 78955.34 & & $9.807[5]$ & $4.56[-1]^{a}$ & $4.55[-1]$ & 0.145 \\
\hline & $\rightarrow 6 p_{1 / 2}$ & 78432.68 & & $4.942[5]$ & $4.54[-1]^{a}$ & $4.53[-1]$ & 0.073 \\
\hline & $\rightarrow 5 p_{3 / 2}$ & 18004.69 & & $1.140[6]$ & $2.70[-2]^{a}$ & $2.75[-2]$ & 0.168 \\
\hline & $\rightarrow 5 p_{1 / 2}$ & 17944.11 & & $5.770[5]$ & $2.71[-2]^{a}$ & $2.77[-2]$ & 0.085 \\
\hline & $\rightarrow 4 p_{3 / 2}$ & 5803.37 & $2.35[6]^{b}$ & $2.375[6]$ & $5.68[-3]^{a}, 5.93[-3]^{b}$ & $5.96[-3]$ & 0.351 \\
\hline & $\rightarrow 4 p_{1 / 2}$ & 5784.00 & $1.19[6]^{b}$ & $1.201[6]$ & $5.71[-3]^{a}, 5.95[-3]^{b}$ & $5.99[-3]$ & 0.177 \\
\hline \multirow[t]{3}{*}{$5 f_{7 / 2}$} & $\rightarrow 5 d_{5 / 2}$ & 237255.75 & & $6.019[4]$ & $6.87[-1]^{a}$ & $6.73[-1]$ & 0.007 \\
\hline & $\rightarrow 4 d_{5 / 2}$ & 31156.02 & & $2.101[6]$ & $3.93[-1]^{a}$ & $4.05[-1]$ & 0.239 \\
\hline & $\rightarrow 3 d_{5 / 2}$ & 11022.87 & $6.54[6]^{b}$ & $6.612[6]$ & $1.76[-1]^{a}, 1.59[-1]^{b}$ & $1.60[-1]$ & 0.754 \\
\hline \multirow[t]{6}{*}{$5 f_{5 / 2}$} & $\rightarrow 5 d_{3 / 2}$ & 237539.62 & & $5.593[4]$ & $7.20[-1]^{a}$ & $7.05[-1]$ & 0.006 \\
\hline & $\rightarrow 5 d_{5 / 2}$ & 237255.75 & & $4.012[3]$ & $3.43[-2]^{a}$ & $3.36[-2]$ & 0.0004 \\
\hline & $\rightarrow 4 d_{3 / 2}$ & 31166.41 & & $1.954[6]$ & $4.13[-1]^{a}$ & $4.24[-1]$ & 0.223 \\
\hline & $\rightarrow 4 d_{5 / 2}$ & 31156.02 & & $1.400[5]$ & $1.97[-2]^{a}$ & $2.02[-2]$ & 0.016 \\
\hline & $\rightarrow 3 d_{3 / 2}$ & 11025.67 & $6.10[6]^{b}$ & $6.171[6]$ & $1.84[-1]^{a}, 1.67[-1]^{b}$ & $1.68[-1]$ & 0.734 \\
\hline & $\rightarrow 3 d_{5 / 2}$ & 11022.87 & $4.36[5]^{b}$ & $4.406[5]$ & $8.78[-3]^{a}, 7.94[-3]^{b}$ & $7.98[-3]$ & 0.050 \\
\hline \multirow[t]{4}{*}{$5 g_{7 / 2}$} & $\rightarrow 5 f_{5 / 2}$ & 9451795.84 & & 0.524 & & $9.30[-3]$ & $\sim 0$ \\
\hline & $\rightarrow 5 f_{7 / 2}$ & 9451795.84 & & 0.020 & & $2.58[-4]$ & $\sim 0$ \\
\hline & $\rightarrow 4 f_{7 / 2}$ & 40169.35 & & $1.532[5]$ & $3.70[-2]^{a}$ & $3.68[-2]$ & 0.036 \\
\hline & $\rightarrow 4 f_{5 / 2}$ & 40169.35 & & $4.136[6]$ & $1.340^{a}$ & 1.326 & 0.964 \\
\hline \multirow[t]{2}{*}{$5 g_{9 / 2}$} & $\rightarrow 5 f_{7 / 2}$ & 9451795.84 & & 0.543 & & $9.04[-3]$ & $\sim 0$ \\
\hline & $\rightarrow 4 f_{7 / 2}$ & 40169.35 & & $4.289[6]$ & $1.300^{a}$ & 1.289 & 1.000 \\
\hline \multirow[t]{7}{*}{$7 p_{1 / 2}$} & $\rightarrow 7 s_{1 / 2}$ & 125683.20 & & $3.343[5]$ & $7.87[-1]^{a}$ & $7.87[-1]$ & 0.211 \\
\hline & $\rightarrow 5 d_{3 / 2}$ & 113102.67 & & $4.187[5]$ & $3.88[-1]^{a}$ & $3.99[-1]$ & 0.265 \\
\hline & $\rightarrow 6 s_{1 / 2}$ & 27630.49 & & $1.446[5]$ & $1.60[-2]^{a}$ & $1.64[-2]$ & 0.091 \\
\hline & $\rightarrow 4 d_{3 / 2}$ & 27234.95 & & $2.946[5]$ & $1.70[-2]^{a}$ & $1.63[-2]$ & 0.186 \\
\hline & $\rightarrow 3 d_{3 / 2}$ & 10489.97 & $2.17[5]^{b}$ & $2.194[5]$ & $1.93[-3]^{a}, 1.79[-3]^{b}$ & $1.80[-3]$ & 0.139 \\
\hline & $\rightarrow 5 s_{1 / 2}$ & 9956.84 & & $1.193[5]$ & $1.66[-3]^{a}$ & $1.76[-3]$ & 0.075 \\
\hline & $\rightarrow 4 s_{1 / 2}$ & 3218.55 & & $5.108[4]$ & $5.37[-5]^{a}$ & $7.88[-5]$ & 0.003 \\
\hline \multirow[t]{10}{*}{$7 p_{3 / 2}$} & $\rightarrow 7 s_{1 / 2}$ & 124976.36 & & $3.383[5]$ & $1.570^{a}$ & 1.575 & 0.201 \\
\hline & $\rightarrow 5 d_{3 / 2}$ & 112529.94 & & $4.212[4]$ & $7.74[-2]^{a}$ & $7.95[-2]$ & 0.025 \\
\hline & $\rightarrow 5 d_{5 / 2}$ & 112466.19 & & $3.805[5]$ & $4.64[-1]^{a}$ & $4.78[-1]$ & 0.227 \\
\hline & $\rightarrow 6 s_{1 / 2}$ & 27596.18 & & $1.508[5]$ & $3.30[-2]^{a}$ & $3.42[-2]$ & 0.090 \\
\hline & $\rightarrow 4 d_{3 / 2}$ & 27201.61 & & $2.984[4]$ & $3.44[-3]^{a}$ & $3.29[-3]$ & 0.018 \\
\hline & $\rightarrow 4 d_{5 / 2}$ & 27193.69 & & $2.675[5]$ & $2.06[-2]^{a}$ & $1.96[-2]$ & 0.159 \\
\hline & $\rightarrow 3 d_{3 / 2}$ & 10485.02 & $2.21[4]^{b}$ & $2.225[4]$ & $3.92[-4]^{a}, 3.64[-4]^{b}$ & $3.64[-4]$ & 0.013 \\
\hline & $\rightarrow 3 d_{5 / 2}$ & 10482.49 & $1.99[4]^{b}$ & $2.580[5]$ & $2.35[-3]^{a}, 2.18[-3]^{b}$ & $2.19[-3]$ & 0.154 \\
\hline & $\rightarrow 5 s_{1 / 2}$ & 9952.38 & & $1.279[5]$ & $3.57[-3]^{a}$ & $3.78[-3]$ & 0.076 \\
\hline & $\rightarrow 4 s_{1 / 2}$ & 3218.08 & & $6.187[4]$ & $1.36[-4]^{a}$ & $1.92[-4]$ & 0.037 \\
\hline \multirow[t]{8}{*}{$6 d_{5 / 2}$} & $\rightarrow 7 p_{3 / 2}$ & 160990.92 & & $2.755[5]$ & & 1.594 & 0.293 \\
\hline & $\rightarrow 5 f_{5 / 2}$ & 91812.99 & & $7.227[3]$ & & $9.08[-3]$ & 0.008 \\
\hline & $\rightarrow 5 f_{7 / 2}$ & 91812.99 & & $1.446[5]$ & & $1.36[-1]$ & 0.154 \\
\hline & $\rightarrow 6 p_{3 / 2}$ & 37199.74 & & $5.876[4]$ & & $1.82[-2]$ & 0.063 \\
\hline & $\rightarrow 4 f_{5 / 2}$ & 28026.51 & & $4.489[3]$ & & $5.25[-4]$ & 0.005 \\
\hline & $\rightarrow 4 f_{7 / 2}$ & 28026.51 & & $8.991[4]$ & & $7.89[-3]$ & 0.096 \\
\hline & $\rightarrow 5 p_{3 / 2}$ & 14335.35 & & $2.321[2]$ & & $1.07[-5]$ & 0.0002 \\
\hline & $\rightarrow 4 p_{3 / 2}$ & 5361.07 & $4.86[5]^{b}$ & $3.577[5]$ & $2.81[-3]^{a}, 3.14[-3]^{b}$ & $2.30[-3]$ & 0.004 \\
\hline \multirow[t]{8}{*}{$6 d_{3 / 2}$} & $\rightarrow 7 p_{3 / 2}$ & 160832.18 & & $4.597[4]$ & & $1.77[-1]$ & 0.049 \\
\hline & $\rightarrow 7 p_{1 / 2}$ & 159676.53 & & $2.333[5]$ & & 1.773 & 0.250 \\
\hline & $\rightarrow 5 f_{5 / 2}$ & 91790.61 & & $1.517[5]$ & & $1.27[-1]$ & 0.162 \\
\hline & $\rightarrow 6 p_{3 / 2}$ & 37196.07 & & $9.882[3]$ & & $2.04[-3]$ & 0.011 \\
\hline & $\rightarrow 6 p_{1 / 2}$ & 37079.66 & & $5.274[4]$ & & $2.16[-2]$ & 0.056 \\
\hline & $\rightarrow 4 f_{5 / 2}$ & 28024.42 & & $9.475[4]$ & & $7.40[-3]$ & 0.101 \\
\hline & $\rightarrow 5 p_{3 / 2}$ & 14334.81 & & 38.69 & & $1.18[-6]$ & $\sim 0$ \\
\hline & $\rightarrow 5 p_{1 / 2}$ & 14296.37 & & $6.034[2]$ & & $3.67[-5]$ & 0.0006 \\
\hline
\end{tabular}


TABLE III - continued from previous page.

\begin{tabular}{|c|c|c|c|c|c|c|c|}
\hline \multirow{2}{*}{$\begin{array}{l}\text { Upper } \\
\text { state }(f)\end{array}$} & \multirow{2}{*}{$\begin{array}{c}\text { Lower } \\
\text { state }(i) \\
\end{array}$} & \multirow[t]{2}{*}{$\lambda_{k i}$} & \multicolumn{2}{|c|}{$A_{k i}$} & \multicolumn{2}{|l|}{$f_{k i}$} & \multirow{2}{*}{$\begin{array}{c}\Gamma_{k i} \\
\text { Present } \\
\end{array}$} \\
\hline & & & Others & Present & Others & Present & \\
\hline \multirow{10}{*}{$8 s_{1 / 2}$} & $\rightarrow 4 p_{3 / 2}$ & 5360.99 & $8.06[4]^{b}$ & $5.991[4]$ & $3.10[-4]^{a}, 3.47[-4]^{b}$ & $2.56[-4]$ & 0.064 \\
\hline & $\rightarrow 4 p_{1 / 2}$ & 5344.45 & $3.86[5]^{b}$ & $2.849[5]$ & $2.96[-3]^{a}, 3.30[-3]^{b}$ & $2.42[-3]$ & 0.305 \\
\hline & $\rightarrow 7 p_{3 / 2}$ & 144722.68 & & $3.779[5]$ & $5.93[-1]^{a}$ & $5.90[-1]$ & 0.093 \\
\hline & $\rightarrow 7 p_{1 / 2}$ & 143786.27 & & $1.905[5]$ & $5.91[-1]^{a}$ & $5.87[-1]$ & 0.047 \\
\hline & $\rightarrow 6 p_{3 / 2}$ & 36262.54 & & $3.760[5]$ & $3.60[-2]^{a}$ & $3.68[-2]$ & 0.093 \\
\hline & $\rightarrow 6 p_{1 / 2}$ & 36151.89 & & $1.906[5]$ & $3.60[-2]^{a}$ & $3.71[-2]$ & 0.047 \\
\hline & $\rightarrow 5 p_{3 / 2}$ & 14193.98 & & $6.079[5]$ & $8.77[-3]^{a}$ & $9.12[-3]$ & 0.150 \\
\hline & $\rightarrow 5 p_{1 / 2}$ & 14156.31 & & $3.009[5]$ & $8.84[-3]^{a}$ & $8.98[-3]$ & 0.074 \\
\hline & $\rightarrow 4 p_{3 / 2}$ & 5341.17 & $1.31[6]^{b}$ & $1.328[6]$ & $2.70[-3]^{a}, 2.81[-3]^{b}$ & $2.82[-3]$ & 0.328 \\
\hline & $\rightarrow 4 p_{1 / 2}$ & 532.481 & $6.64[5]^{b}$ & $6.700[5]$ & $2.71[-3]^{a}, 2.82[-3]^{b}$ & $2.83[-3]$ & 0.166 \\
\hline
\end{tabular}

References: ${ }^{a}$ 32]; ${ }^{b} 33$

\section{B. E1 matrix elements and oscillator strengths}

We present E1 matrix elements from the DHF and CCSD(T) methods along with their line strengths from latter in Table [I]. We estimate uncertainties associated with the calculations using the $\operatorname{CCSD}(\mathrm{T})$ method as both DHF and CCSD methods are just part of this approach. To estimate net uncertainty of an E1 matrix element, we take into account incompleteness of basis functions and differences in the results obtained using the CCSD and $\operatorname{CCSD}(\mathrm{T})$ methods to estimate uncertainty due to the approximation in the level of excitations. We also find that the correlation contributions, estimated as the differences between the results obtained using the DHF and $\operatorname{CCSD}(\mathrm{T})$ methods, are significant in almost all the transitions.

It is not always possible to obtain precise values of either E1 matrix elements or transition strengths from the measured lifetimes due to association of many transition probabilities with these quantities. However, the $4 P$ states are the first two excited states which decay to the ground state only via one allowed transition each. There are precise measurements of lifetimes of these states are available in $\mathrm{K}$ atom. The lifetime of the $4 P_{1 / 2}$ state is reported to be 26.69(5) ns 44]. By combining this result with the experimental value of wavelength $\lambda=7701.1$ $\AA$ [28] of the $4 P_{1 / 2} \rightarrow 4 S$ transition, we find the E1 matrix element of the $4 P_{1 / 2} \rightarrow 4 S$ transition to be $4.110(5)$ a.u. against our calculated result $4.131(20)$ a.u. Similarly, the lifetime of the $4 P_{3 / 2}$ state is measured to be 26.34(5) ns [44]. This state has an allowed transition channel to the ground state and it can also decay to the first excited $4 P_{1 / 2}$ state via both the electric quadrupole (E2) and magnetic dipole (M1) channels. It is found from our analysis that the transition probabilities of an electron due to the above forbidden channels from the $4 P_{3 / 2}$ state are very small and can be neglected within our estimated uncertainties. Therefore combining the measured lifetime of the $4 P_{3 / 2}$ state with the experimental value of the $4 P_{3 / 2} \rightarrow 4 S$ transition wavelength $\lambda=7667.0 \AA$, we
TABLE IV: Lifetimes $(\tau)$ of all the low-lying states up to $8 S$ in $\mathrm{K}$ atom (in $n s$ ).

\begin{tabular}{|c|c|c|c|}
\hline State & This work & Others & Experiment \\
\hline $5 s^{2} S_{1 / 2}$ & $42.31(1.1)$ & $46.50^{a}, 42.5^{b}$ & \\
\hline $3 d^{2} D_{5 / 2}$ & $41.85(1.2)$ & $45.85^{a}, 42.5^{b}$ & \\
\hline $3 d^{2} D_{3 / 2}$ & $41.32(1.0)$ & $45.24^{a}, 41.9^{b}$ & $42(3)^{g}, 42(3)^{d}$ \\
\hline $5 p^{2} P_{1 / 2}$ & $134.40(3.0)$ & $\begin{array}{c}127.05^{a}, 137.1^{b} \\
130^{h}\end{array}$ & $137.6(1.3)^{k}$ \\
\hline $5 p^{2} P_{3 / 2}$ & $131.10(3.0)$ & $124.02^{a}, 133.9^{b}$ & $133(3)^{f}, 134(2)^{e}$ \\
\hline $4 d^{2} D_{5 / 2}$ & $290.02(8.0)$ & $291.18^{a}, 293.9^{b}$ & \\
\hline $4 d^{2} D_{3 / 2}$ & $284.10(7.3)$ & $285.56^{a}, 289.4^{b}$ & \\
\hline $6 s^{2} S_{1 / 2}$ & $80.81(1.1)$ & $87.12^{a}, 81.4^{b}$ & $88(2)^{d}, 68(9)^{i}$ \\
\hline $4 f^{2} F_{5 / 2}$ & $62.30(2.0)$ & $70.65^{a}, 64.7^{b}$ & \\
\hline $4 f^{2} F_{7 / 2}$ & $62.20(2.0)$ & $70.65^{a}, 63.9^{b}$ & \\
\hline $6 p^{2} P_{1 / 2}$ & $332.70(8.2)$ & $321.67^{a}, 340.7^{b}$ & $344(3)^{e}$ \\
\hline $6 p^{2} P_{3 / 2}$ & $324.20(7.1)$ & $312.77^{a}, 332.0^{b}$ & $333(3)^{e}, 310(15)^{f}$ \\
\hline $5 d^{2} D_{5 / 2}$ & $713.70(19.1)$ & $769.63^{a}, 650.8^{b}$ & \\
\hline $5 d^{2} D_{3 / 2}$ & $712.70(21.1)$ & $767.41^{a}, 653.1^{b}$ & $572(14)^{d}, 610(90)^{j}$ \\
\hline $7 s^{2} S_{1 / 2}$ & $147.71(1.2)$ & $158.83^{a}, 148.8^{b}$ & $155(6)^{d}, 165(12)^{j}$ \\
\hline $5 f^{2} F_{7 / 2}$ & $114.11(3.2)$ & $125.70^{a}, 117.9^{b}$ & \\
\hline $5 f^{2} F_{5 / 2}$ & $114.12(3.1)$ & $125.70^{a}, 118.0^{b}$ & $117(3)^{c}$ \\
\hline $5 g^{2} G_{7 / 2}$ & $233.20(1.3)$ & & \\
\hline $5 g^{2} G_{9 / 2}$ & $233.20(1.3)$ & & \\
\hline $7 p^{2} P_{1 / 2}$ & $632.10(15.2)$ & $619.47^{a}, 648.6^{b}$ & $623(6)^{e}$ \\
\hline $7 p^{2} P_{3 / 2}$ & $595.50(14.1)$ & $601.80^{a}, 632.0^{b}$ & $592(6)^{e}$ \\
\hline $6 d^{2} D_{5 / 2}$ & $1066.10(24.1)$ & $1168.54^{b}, 913.7^{a}$ & \\
\hline $6 d^{2} D_{3 / 2}$ & $1071.11(26.0)$ & $\begin{array}{c}1180.58^{a}, 925.7^{b} \\
1050^{h}\end{array}$ & $807(20)^{d}, 890(60)^{j}$ \\
\hline $8 s^{2} S_{1 / 2}$ & $247.40(2.0)$ & $267.23^{a}, 250.7^{b}$ & $238(4)^{d}, 260(14)^{j}$ \\
\hline
\end{tabular}

obtain E1 matrix element of the $4 P_{3 / 2} \rightarrow 4 S$ transition as 5.812(6) a.u. Although our calculated results from the $\operatorname{CCSD}(\mathrm{T})$ method are in agreement with the extracted values from the experimental lifetimes of the $4 P$ states, however we consider below the precise values obtained from the measurements to evaluate polarizabilities.

For the astrophysical interest, we also evaluate transition probabilities, oscillator strengths for emission lines and branching ratios taking all possible allowed transi- 
TABLE V: Scalar polarizability of the ground state in a.u. in $\mathrm{K}$ along with individual contribution from various intermediate terms. Uncertainties are quoted in the parentheses.

\begin{tabular}{rr}
\hline \hline \multicolumn{2}{c}{ Contribution } \\
\hline \multicolumn{4}{c}{$\alpha_{v} \quad \alpha_{v}^{(0)}$} \\
$4 p_{1 / 2}$ & $95.165(231)$ \\
$4 p_{3 / 2}$ & $189.471(391)$ \\
$5 p_{1 / 2}$ & $0.235(10)$ \\
$5 p_{3 / 2}$ & $0.514(10)$ \\
$6 p_{1 / 2}$ & $0.019(3)$ \\
$6 p_{3 / 2}$ & $0.044(3)$ \\
$7 p_{1 / 2}$ & $0.004(3)$ \\
$7 p_{3 / 2}$ & $0.010(3)$ \\
$8 p_{1 / 2}$ & $0.001(3)$ \\
$8 p_{3 / 2}$ & $0.003(2)$ \\
$9 p_{1 / 2}$ & $0.001(2)$ \\
$9 p_{3 / 2}$ & $0.002(2)$ \\
$\alpha_{c}$ & $5.131(0.30)$ \\
$\alpha_{c n}$ & $-0.127(0.02)$ \\
$\alpha_{\text {tail }}$ & $0.062(0.01)$ \\
$\alpha_{\text {total }}$ & $290.5(1.0)$ \\
Experiment & $292.8(6.1)[45], 305.0(21.6)[46]$ \\
Others & $290.58(1.42)[47]$ \\
\hline \hline
\end{tabular}

tions among the considered states in $\mathrm{K}$ atom using the above E1 matrix elements and tabulated wavelengths in NIST [28]. These values are given in Table [II] Four decade ago, Anderson and Ziltis had carried out calculations of these quantities using a Coulomb approximation in the non-relativistic method [49]. Safronova and Safronova have also reported few of these quantities using a linearized approximation to the CCSD method (SD method) 33. In a recent paper, Civiš et al have used a quantum defect theory to evaluate these quantities in many transitions which are of particular astrophysical interest and compared with the estimated results from various measurements and observations [32] including those are given in [49]. In the above table, we compare our results along with the values reported in [32, 33]. As seen in the table, all the results are in fair agreement among each other. Many of these results related to the $6 D$ states were not known previously to compare with the present estimation. We also give branching ratios explicitly of individual allowed transition in the same table by considering into account all possible allowed transitions and neglecting transition probabilities due to the forbidden transitions.

\section{Lifetimes of few excited states}

As mentioned above, the lifetimes of the $4 P$ states in $\mathrm{K}$ atom are measured precisely by Wang et al [44]. However, these quantities are not measured for some of the low-lying states. In fact, many measured values available
TABLE VI: Scalar polarizability of the $4 P_{1 / 2}$ state of $\mathrm{K}$ atom in a.u. Uncertainties are given in the parentheses.

\begin{tabular}{|c|c|c|}
\hline \multicolumn{2}{|c|}{ Contribution } & $\alpha_{v}^{(0)}$ \\
\hline \multicolumn{3}{|c|}{$\alpha_{v}$} \\
\hline $4 p_{1 / 2} \rightarrow$ & $4 s_{1 / 2}$ & $-95.165(231)$ \\
\hline & $5 s_{1 / 2}$ & $136.679(705)$ \\
\hline & $6 s_{1 / 2}$ & $4.179(100)$ \\
\hline & $7 s_{1 / 2}$ & $0.971(20)$ \\
\hline & $8 s_{1 / 2}$ & $0.389(10)$ \\
\hline & $9 s_{1 / 2}$ & $0.188(10)$ \\
\hline & $10 s_{1 / 2}$ & $0.003(5)$ \\
\hline & $3 d_{3 / 2}$ & $545.86(683)$ \\
\hline & $4 d_{1 / 2}$ & $0.246(8)$ \\
\hline & $5 d_{3 / 2}$ & $0.296(10)$ \\
\hline & $6 d_{3 / 2}$ & $0.336(10)$ \\
\hline & $7 d_{3 / 2}$ & $0.017(5)$ \\
\hline & $8 d_{3 / 2}$ & $0.009(5)$ \\
\hline & & $5.131(0.30)$ \\
\hline$\alpha$ & & $-0.0002(0.001)$ \\
\hline$\alpha_{\mathrm{t}}$ & & $6.4(2.0)$ \\
\hline$\alpha_{\mathrm{t}}$ & & $606(7)$ \\
\hline Exper & ent & $587(87)[50]$ \\
\hline Otl & & 604.1 [33], 602(11)[7], 697.4 [51] \\
\hline
\end{tabular}

for the states other than the $4 P$ states are not very precise. Using our calculated transition probabilities given in Table III, we determine lifetimes of all the considered states except for the $4 P$ states. These results are given in Table IV and compared with other theoretical and experimental data in the same table. We have also estimated uncertainties to our estimated lifetime values from the reported error bars of E1 matrix elements. Most of our results are within the error bars of the experimental values. It can be seen in the above table that the present values of lifetimes of many low-lying states match well with the other theoretical results given in [33] and differ from another calculated results reported in [34]; but our results for some of the higher states match better with the values given in [34] than the results given in [33]. Lifetimes of the $5 G$ states were not found elsewhere to compare with our results.

\section{Static dipole polarizabilities $(\omega=0)$}

We evaluate static dipole polarizabilities of the ground, $4 P$ and $3 D$ states in $\mathrm{K}$ atom using the reported $\mathrm{E} 1$ matrix elements and experimental energies of the most important intermediate states. These results are reported in Tables $\mathrm{V}$ to $\mathrm{IX}$ along with individual contributions explicitly from various intermediate states and compare them with other available experimental and calculations using both non-relativistic and relativistic methods. As has been stated earlier, the core and core-valence correlation contributions are estimated using the $\operatorname{MBPT}(3)$ 
TABLE VII: Contributions to both the scalar and tensor polarizabilities of the $4 P_{3 / 2}$ state in $\mathrm{K}$ atom in a.u. Uncertainties are mentioned in the parentheses.

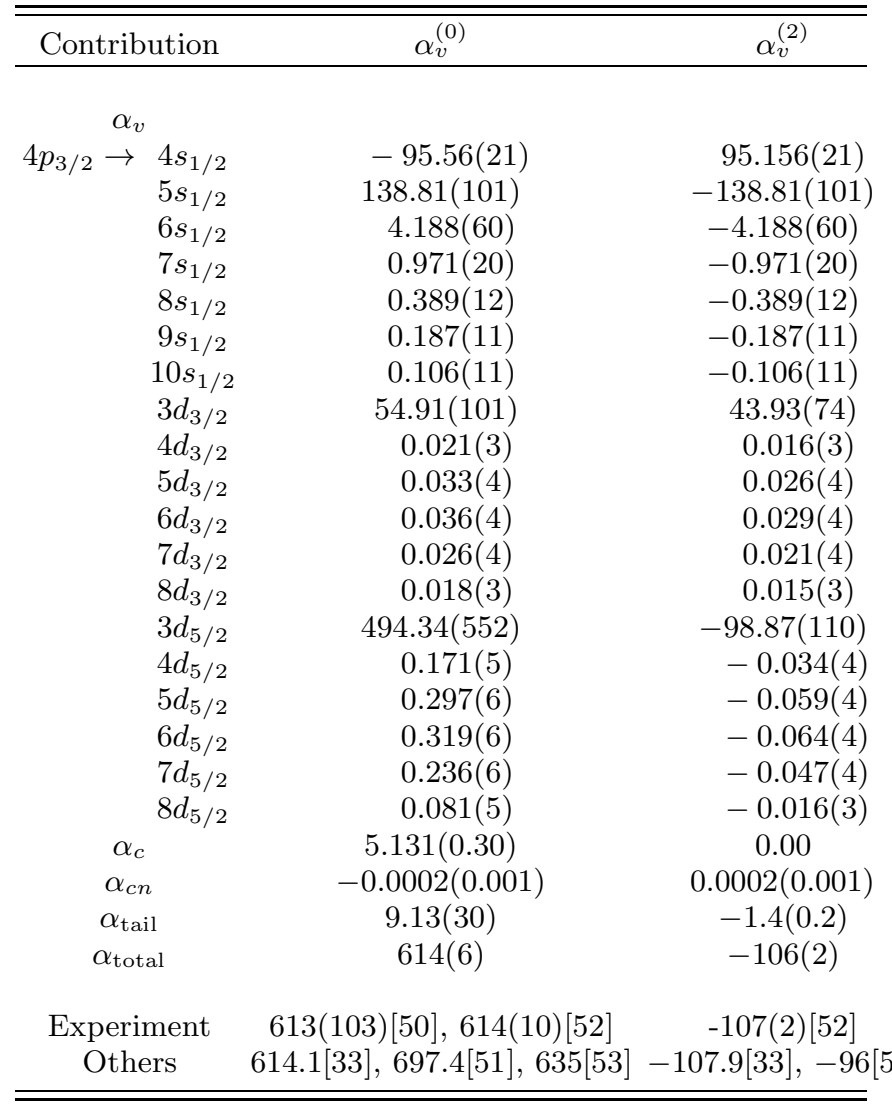

method. Since these contributions are relatively smaller than the valence correlation contribution, the accuracies of the final results are uninfluenced by these results. In fact, we also give estimated uncertainties to these results by comparing E1 matrix elements obtained using this method with the $\operatorname{CCSD}(\mathrm{T})$ method. It can be noticed from these result tables that only few studies are carried out on polarizabilities in $\mathrm{K}$. Also, the experimental results from the direct measurements are not available precisely in any of the considered states. Recently, there is a result on the ground state polarizability in $\mathrm{K}$ has been reported by combining the measured ground state polarizability of sodium $(\mathrm{Na})$ atom with the ratio of these quantities between $\mathrm{K}$ and $\mathrm{Na}$ and reported as 290.58(1.42) a.u. [47]. Similarly, the differential polarizability of the $4 P_{1 / 2} \rightarrow 4 S$ transition has been measured to be 317.11(4) a.u. [54]. By combining this result with the above ground state value, we refer the experimental value of the $4 P_{1 / 2}$ state polarizability as $607.69(2.97)$ a.u. which seem to be in excellent agreement with our calculated result. Other previous calculations reporting this value in [7, 12, 33, 51] are based on methods using lower approximations than the present work. There are no experimental results in the $3 D$ states available to compare the corresponding results with our calculations.
TABLE VIII: Scalar and tensor polarizabilities of the $3 D_{3 / 2}$ state along with uncertainties in the parentheses given in a.u.

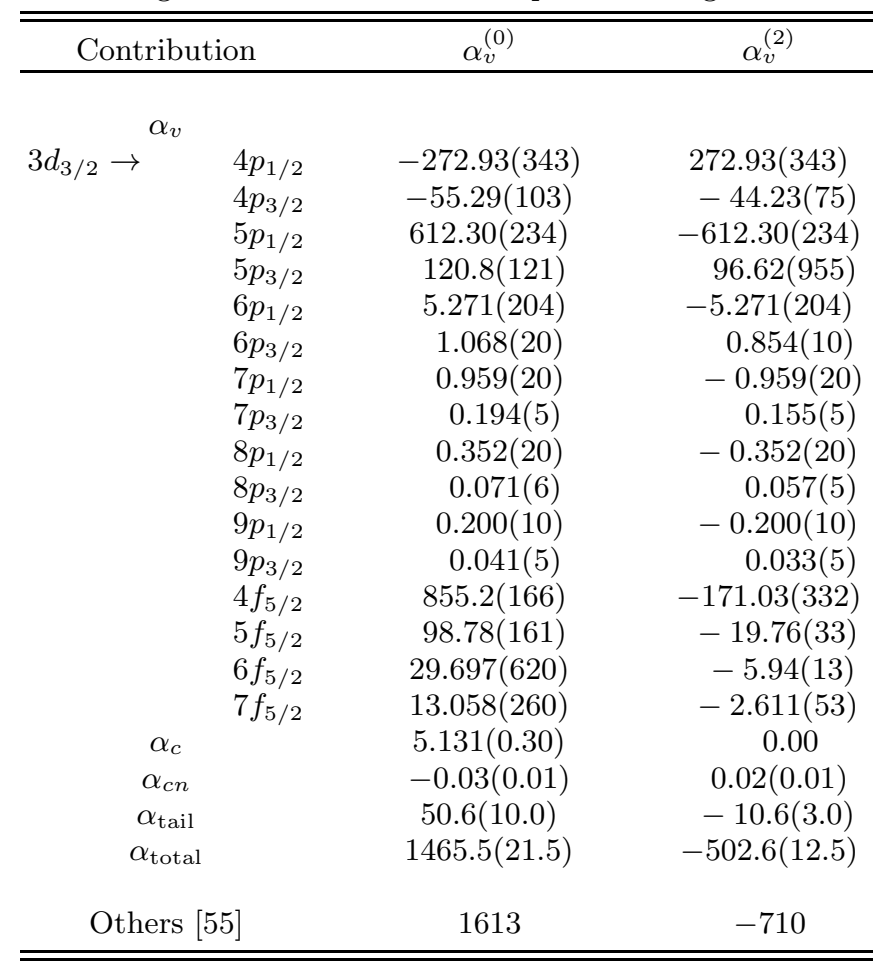

However, a calculation in the non-relativistic method using a pseudo-potential is reported on both the scalar and tensor polarizabilities of the $3 D$ states [55]. These values are also given in Tables VIII and IX, which also seem to be fairly agreement with our calculations.

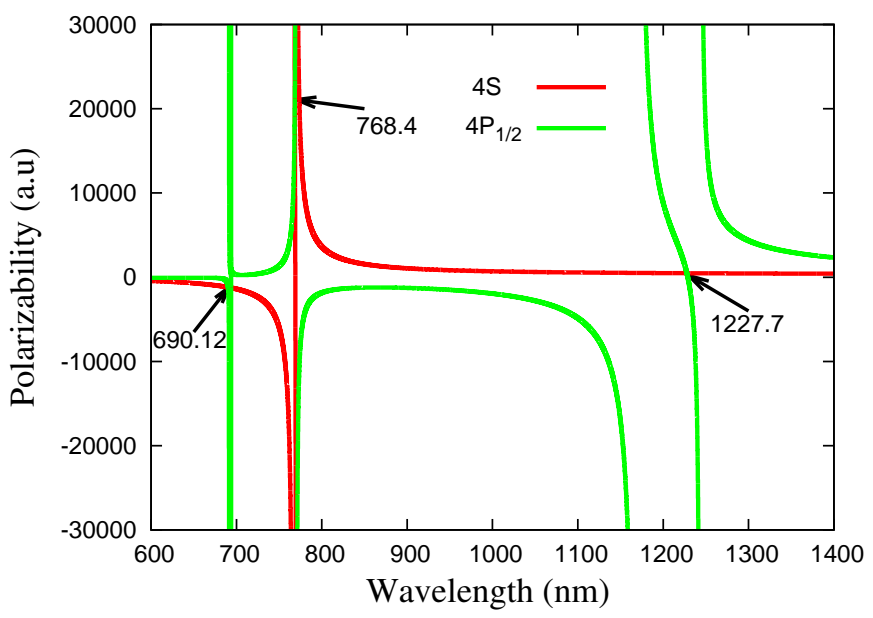

FIG. 1: (color online) Dynamic polarizabilities (in a.u.) plots of the $4 S$ (shown in red) and $4 P_{1 / 2}$ (shown in green) states against wavelengths (in $\mathrm{nm}$ ). Intersection points are identified as magic wavelengths of the $4 P_{1 / 2}-4 S$ transition. Resonance lines correspond at infinite values of polarizabilities. 
TABLE IX: Contributions to both the scalar and tensor polarizabilities of the $3 D_{5 / 2}$ state in $\mathrm{K}$ (in a.u.) with their uncertainties in the parentheses.

\begin{tabular}{|c|c|c|c|}
\hline \multicolumn{2}{|c|}{ Contribution } & $\alpha_{v}^{(0)}$ & $\alpha_{v}^{(2)}$ \\
\hline \multicolumn{4}{|c|}{$\alpha_{v}$} \\
\hline $3 d_{5 / 2} \rightarrow$ & $4 p_{3 / 2}$ & $-331.79(371)$ & $331.79(371)$ \\
\hline & $5 p_{3 / 2}$ & $724.7(240)$ & $-724.7(239)$ \\
\hline & $6 p_{3 / 2}$ & $6.378(182)$ & $-6.378(182)$ \\
\hline & $7 p_{3 / 2}$ & $1.168(20)$ & $-1.168(20)$ \\
\hline & $8 p_{3 / 2}$ & $0.425(15)$ & $-0.425(15)$ \\
\hline & $9 p_{3 / 2}$ & $0.246(12)$ & $-0.246(12)$ \\
\hline & $4 f_{5 / 2}$ & $40.7(101)$ & $46.54(112)$ \\
\hline & $4 f_{7 / 2}$ & $814.7(144)$ & $-290.96(512)$ \\
\hline & $5 f_{5 / 2}$ & $4.698(140)$ & $5.369(160)$ \\
\hline & $5 f_{7 / 2}$ & $93.99(161)$ & $-33.57(58)$ \\
\hline & $6 f_{5 / 2}$ & $1.413(20)$ & $1.615(20)$ \\
\hline & $6 f_{7 / 2}$ & $27.94(485)$ & $-9.98(173)$ \\
\hline & $7 f_{5 / 2}$ & $0.178(5)$ & $0.203(5)$ \\
\hline & $7 f_{7 / 2}$ & $12.36(23)$ & $-4.42(12)$ \\
\hline$\alpha$ & & $5.131(0.30)$ & 0.0 \\
\hline$\alpha_{c}$ & & $-0.03(0.01)$ & $0.03(0.01)$ \\
\hline$\alpha_{\mathrm{t}}$ & & $50.6(10.0)$ & $-15.4(4.0)$ \\
\hline$\alpha_{\mathrm{to}}$ & & $1452.8(32.5)$ & $-701.7(25.6)$ \\
\hline Oth & & $1613[55]$ & $-710[55]$ \\
\hline
\end{tabular}

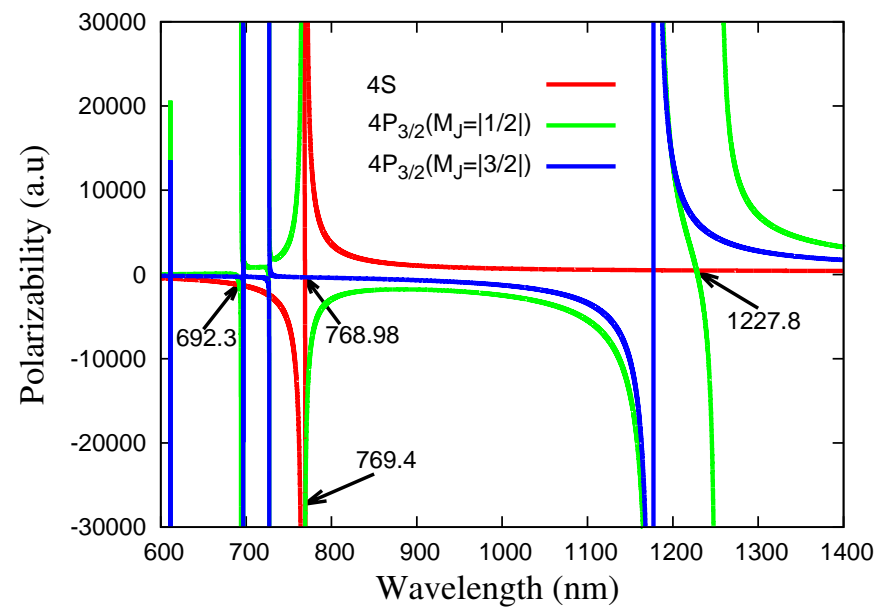

FIG. 2: (color online) Dynamic polarizabilities (in a.u.) plots of the $4 S$ and $4 P_{3 / 2}$ states with different $M_{J}$ values against wavelengths (in $\mathrm{nm}$ ). Lines shown with red, green and blue colors represent for results of the $4 S$ state, $4 P_{3 / 2}$ state with $M_{J}=1 / 2$ and $4 P_{3 / 2}$ state with $M_{J}=3 / 2$, respectively. Magic wavelengths are shown by arrows.

\section{E. Reexamination of magic wavelengths}

Since our reported static polarizabilities for the ground and $4 P$ states seem to be more accurate than the results reported in [7], we intend here to further study the dynamic polarizabilities and estimate the magic wavelengths in the $4 P_{1 / 2} \rightarrow 4 S$ and $4 P_{3 / 2} \rightarrow 4 S$ transitions
TABLE X: Comparison of magic wavelengths and their corresponding polarizability values around different resonance lines for the $4 P_{1 / 2} \rightarrow 4 S$ transition with other works.

\begin{tabular}{cccccc}
\hline \hline \multirow{2}{*}{$\lambda_{\text {res }}$} & \multicolumn{2}{c}{$\lambda_{\text {magic }}$} & & \multicolumn{2}{c}{$\alpha\left(\lambda_{\text {magic }}\right)$} \\
\cline { 2 - 3 } \cline { 5 - 5 } 1243.57 & Present & Ref. $[7]$ & & Present & Ref. [7] \\
\hline 1169.34 & & & & \\
770.11 & & & & & \\
& $768.412(3)$ & $768.413(4)$ & $21072(1)$ & \\
& & & & \\
693.30 & & & & \\
691.12 & & & & \\
& $690.12(2)$ & $690.15(1)$ & $-1190(3)$ & $-1186(2)$ \\
\hline \hline
\end{tabular}

to compare them with the values given in [7]. In Fig. 1. we plot the dynamic polarizabilities of both the $4 S$ and $4 P_{1 / 2}$ states and look for the intersections outside the resonance lines. From this plot, we give the magic wavelengths corresponding to the intersection points of polarizabilities from both the states. The polarizability values and magic wavelengths around different resonance lines for the $4 P_{1 / 2} \rightarrow 4 S$ transition are given in Table $\mathrm{X}$. From the analysis of the above plot, we compare our results with the ones given in [7]. We also find same number of magic wavelengths as in [7], but the corresponding values are further fine-tuned in this work due to more accurate values of the polarizabilities. We also find similar results for the $4 P_{3 / 2} \rightarrow 4 S$ transition and they are given in Table [X] As pointed out in [7] and also found in the present work, there is only one magic wavelength found for the $M_{J}=3 / 2$ sublevel of the $4 P_{3 / 2}$ state. It has to be noted that for the linearly polarized light, the Stark shift of an energy level is independent of the sign of the $M_{J}$ value. However, this shift depends on the sign of the $M_{J}$ value in the circularly polarized light due to the presence of the vector component of the polarizability. It is found in our another recent study in rubidium (Rb) atom [8] that it is possible to obtain more number of magic wavelengths using the circularly polarized light than the linearly polarized light and we also anticipate for similar results in the $4 P_{3 / 2} \rightarrow 4 S$ transition in $\mathrm{K}$ using the circularly polarized light.

\section{CONCLUSION}

We have studied electron attachment energies, electric dipole matrix elements, oscillator strengths, lifetimes and dipole polarizabilities in potassium atom. Some of the reported results are improved significantly than the previously known results. We affirm the reported magic wavelengths in the considered atom using the dynamic polarizabilities. Our oscillator strength results will be very useful in the astrophysical studies and other improved results will be very helpful in guiding the future 
TABLE XI: Magic wavelengths and their corresponding dynamic polarizabilities for the $4 P_{3 / 2} \rightarrow 4 S$ transition with $M_{J}=1 / 2$ and $M_{J}=3 / 2$ values from different works.

\begin{tabular}{|c|c|c|c|c|}
\hline \multirow[t]{2}{*}{$\lambda_{\text {res }}$} & \multicolumn{2}{|c|}{$\lambda_{\text {magic }}$} & \multicolumn{2}{|c|}{$\alpha\left(\lambda_{\text {magic }}\right)$} \\
\hline & Present & Ref. $[7]$ & Present & Ref. [7] \\
\hline \multicolumn{5}{|c|}{$\left|M_{J_{3 / 2}}\right|=1 / 2$} \\
\hline 1252.56 & $1227.8(2)$ & $1227.7(2)$ & $474(2)$ & $472(1)$ \\
\hline \multicolumn{5}{|l|}{1177.61} \\
\hline \multicolumn{5}{|l|}{1177.29} \\
\hline & $769.43(2)$ & $769.432(2)$ & $-27267(63)$ & $-27190(60)$ \\
\hline \multicolumn{5}{|l|}{766.70} \\
\hline \multicolumn{5}{|l|}{696.66} \\
\hline \multicolumn{5}{|l|}{696.61} \\
\hline \multicolumn{5}{|l|}{694.07} \\
\hline & $692.26(3)$ & $692.32(2)$ & $-1230(3)$ & $-1226(3)$ \\
\hline \multicolumn{5}{|c|}{$\left|M_{J_{3 / 2}}\right|=3 / 2$} \\
\hline \multicolumn{5}{|l|}{1177.61} \\
\hline \multicolumn{5}{|l|}{1177.29} \\
\hline & $100.90(2)$ & $100.900(0)$ & $-500.02(0)$ & $-5.00(0)$ \\
\hline
\end{tabular}

[1] C.-H. Wu, I. Santiago, J. W. Park, P. Ahmadi and M. W. Zwierlein, Phys. Rev. A 84, 011601(R) (2011).

[2] J. Catani, P. Maioli, L. De Sarlo, F. Minardi and M. Inguscio, Phys. Rev A 73, 033415 (2006)

[3] L. Allen and J. H. Eberly, Optical Resonance and TwoLevel Atoms, Dover Publications, 1975.

[4] M. Zwierlein, Cooling and Trapping a Bose-Fermi Mixture of Dilute Atomic Gases, Ph.D. Thesis Submitted to Department of Physics, Massachusetts Institute of Technology, Cambridge, USA (2011).

[5] D. C. McKay, D. Jervis, D. J. Fine, J. W. Simpson-Porco, G. J. A. Edge and J. H. Thywissen, Phys. Rev. A 84, 063420 (2011).

[6] H. Katori, K. Hashiguchi, E. Yu. Il'inova and V. D. Ovsiannikov, Phys. Rev. Lett. 103, 153004 (2009).

[7] B. Arora, M. S. Safronova and C. W. Clark, Phys. Rev. A 76, 052509 (2007).

[8] B. Arora and B. K. Shaoo, Phys. Rev. A 86033416 (2012).

[9] L. Yi, S. Mejri, J. J. McFerran, Y. Le Coq and S. Bize, Phys. Rev. Lett. 106, 073005 (2011).

[10] Z.W. Barber, J. E. Stalnaker, N. D. Lemke, N. Poli, C.W. Oates, T. M. Fortier, S. A. Diddams, L. Hollberg, C.W. Hoytk, A.V. Taichenachev and V. I. Yudin, Phys. Rev. Lett. 100, 103002 (2008).

[11] M. Takamoto, F.-L. Hong, R. Higashi and H. Katori, Nature 435, 321 (2005).

[12] A. Derevianko and H. Katori, Rev. Mod. Phys. 83, 331 (2011).

[13] A. Burrows and M. Volobuyev, Astrophys. J. 583, 985 (2003).

[14] C. J. Zeippen, Astron. Astrophys. 229, 248 (1990).

[15] E. Charro, Z. Curiel and I. Martin, Astron. Astrophys. experiments.

\section{Acknowledgment}

We thank B. Arora for discussions regarding magic wavelengths. The calculations were carried out using PRL 3TFLOP HPC cluster, Ahmedabad.
387, 1146 (2002).

[16] H. W. Zhang, T. Gehren, K. Butler, J. R. Shi, and G. Zhao, Astron. Astrophys. 457, 645 (2006).

[17] A. J. Burgasser, J. D. Kirkpatrick, I. N. Reid, J. Liebert, J. E. Gizis and M. E. Brown, Astrophys. J. 120, 473 (2000).

[18] A. Burrows, A. J. Burgasser, J. D. Kirkpatrick, J. Liebert, J. A. Milsom, D. Sudarsky and I. Hubeny, Astrophys. J. 573, 294 (2002).

[19] A. Burrows, W. B. Hubbard, J. I. Lunine and J. Liebert, Rev. Mod. Phys. 73, 719 (2001).

[20] D. Charbonneau, T. M. Brown, R. W. Noyes and R. L. Gilliland, Astrophys. J. 568, 377 (2002).

[21] S. Johansson, High Resolution Infrared Spectroscopy in Astronomy Proccedings, ed. by H. U. Kaufl, R. Siebenmorgen and A. Moorwood, ESO Astrophysics Symposia (Springer-Verlag Publication, Berlin), Nov. 18-21, 2003.

[22] A. Jorissen, Phys. Scr. T112, 73 (2004).

[23] J. Pickering, R. Blackwell-Whitehead, A. Thorne, M. Ruffoni and C. Homles, Can. J. Phys. 89, 387 (2011).

[24] Y. Lyubchik, H. Jones, Y. Pavlenko et al., Astron. Astrophys. 416, 655 (2004).

[25] R. J. Bartlett and M. Musial, Rev. Mod. Phys. 79, 291 (2007).

[26] Yashpal Singh, D. K. Nandy and B. K. Sahoo, Phys. Rev. A 86, 032509 (2012)

[27] Bindiya Arora, D. K. Nandy and B. K. Sahoo, Phys. Rev. A 85, 012506 (2012)

[28] http://physics.nist.gov/cgi-bin/ASD/energy1.pl

[29] B. K. Sahoo, Chem. Phys. Lett. 448, 1441 (2007)

[30] B. K. Sahoo, B. P. Das and D. Mukherjee, Phys. Rev. A 79052511 (2009)

[31] B. K. Sahoo, P. Mandal and M. Mukherjee, Phys. Rev. 
A 83 030502(R) (2011)

[32] S. Civiš, M. Ferus, P. Kubelik, P. Jelinek and V. E. Chernov, A and A 541 A125(2012)

[33] U. I. Safronova and M. S. Safronova, Phys. Rev. A 78, 052504 (2008).

[34] C. E. Theodosiou, Phys. Rev. A 30, 2881 (1984)

[35] M. Glodz, A. Huzandrov, M. S. Safronova, I. Sydoryk, J. Szonert, and J. Klavins, Phys. Rev. A 77, 022503 (2008)

[36] D. J. Hart and J. B. Atkinson, J. Phy. B 19, 43 (1986)

[37] R. W. Berends, W. Kedzierski, J. B. Atkinson, and L. Krause, Spectrochim. Acta, Part B 43, 1069 (1988)

[38] S. Svanberg, Phys. Scr. 4, 275 (1971)

[39] V. Teppner and P. Zimmermann, Astron. Astrophys. (Germany) 64, 215 (1978)

[40] P. F. Grudzev and V. I. Denisov, Opt. Spektrosk. 52, 15 (1982) [Opt. Spectrosc. (USSR) 52, 8 (1982)]

[41] B. R. Bulos, R. Gupta, and W. Happer, J. Opt. Soc. Am. 66, 426 (1976)

[42] T. F. Gallagher and W. E. Cooke, Phys. Rev. A 20, 670 (1970)

[43] A. Mills, J. A. Behr, L. A. Courneyea, and M. R. Pearson, Phys. Rev. A 72, 024501 (2005)

[44] H. Wang, J. Li, X. T. Wang, C. J. Williams, P. L. Gould, and W. C. Stwalley, Phys. Rev. A 55, R1569 (1997)

[45] R. W. Molof, H. L. Schwartz, T. M. Miller and B. Bederson, Phys. Rev. A 10, 1131 (1974).

[46] W. D. Hall and J. C. Zorn, Phys. Rev. A 10, 1141 (1974).

[47] W. F. Holmgren, M. C. Revelle, V. P. A. Lonij and A. D. Cronin, Phys. Rev. A 81, 053607 (2010).

[48] A. Derevianko, W. R. Johnson, M. S. Safronova and J. F. Babb, Phys. Rev. Lett. 82, 3589 (1999).

[49] E. M. Anderson and V. A. Zilitis, Opt. Spectrosk, 16, 177 (1964).

[50] R. Marrus and J. Yellin, Phys. Rev. 177127 (1969)

[51] M. Mérawa and D. Bégué, J. Chem. Phys. 108, 5289 (1998).

[52] C. Krenn, W. Scherf, O. Khait, M. Musso and L. Windholz, Z. Phys. D; At., Mol. Clusters 41, 229(1997)

[53] R. Schmieder, A. Lurio, and W. Happer, Phys. Rev. A 3, 1209 (1971).

[54] K. E. Miller, D. Krause and L. R. Hunter, Phys. Rev. A 49, 5128 (1994).

[55] M. Rérat, M. Merawa and B. Honvault-Bussery, J. Chem. Phys. 109, 7246 (1998). 